\title{
BREATHERS IN A PINNED MECHANICAL LATTICE
}

\author{
S.C.GREEN ${ }^{\dagger \ddagger}$, C.J.BUDD ${ }^{\dagger \ddagger}$, AND G.W.HUNT ${ }^{\dagger \S}$
}

\begin{abstract}
Discrete breathers are found in a nonlinear one dimensional axially loaded mechanical lattice consisting of rigid links supported laterally by linear springs. We find link centered breathers for an odd number of mechanical links and pivot centred breathers where the number of links is even. Substantial parameter regions in load-frequency parameter space are found where these breathers are linearly and nonlinearly stable. This region includes the lattice in tension, in compression and in the unloaded state. We also find that despite the rigid nature of this mechanical system both the lateral displacement and energy-per-link is, at least, exponentially localised in the breather core. Initial results also point towards the ability of energy to spontaneously localise in this mechanical lattice. This is seen in the form of a spontaneously appearing moving discrete breather.
\end{abstract}

Key words. Mechanical lattice, Mechanical structure, Hamiltonian lattice, Dynamics, Discrete Breathers, Localisation

AMS subject classifications. 00A06 34K13 70E50 70E55 70H12 70J50 70K42

1. Introduction. Pinned and linked mechanical lattice systems of the form illustrated in Figure 1.1 as well as being of great importance in structural engineering [9] have remarkably rich static [5] and dynamic behaviour. In particular, in this paper we show that a mechanical lattice system such as this can support spatially localised, time periodic oscillations, or 'breathers'. Breather solutions are time periodic solutions in a set of coupled ordinary differential equations (ODEs) where the energy is, often exponentially, localised in space (for reviews see $[7,1,2]$ ). Despite the many publications on this topic few of the applications have been from macroscopic engineering systems. Localisation of energy in these systems can have catastrophic consequences, for instance excessive vibration of a single turbine blade leading to premature failure, or vibration concentration in the lattice of a steel building causing excessive fatigue.

A mathematical model for the mechanical system shown in Figure 1.1, derived in detail in $\S 2$ leads to a set of ODEs of the form

$$
(I+A(\mathbf{y})) \ddot{\mathbf{y}}+B(\mathbf{y}, \dot{\mathbf{y}}) \dot{\mathbf{y}}=\mathbf{f}(\mathbf{y})
$$

where $\mathbf{y}$ is the set of vertical pivot displacements and the functions $A, B$ and $\mathbf{f}$ are defined further in $\S 2$. There are several features of this mechanical system and its mathematical model that make the breather investigations in this paper new. Firstly, the rigid mechanical nature of this system means that unlike nearly all other systems that exhibit breather solutions, the vertical displacement of pivots close to the centre of the lattice affect the horizontal movement of the pivots at the ends of the lattice. Thus, even if the vertical displacement of the pivots localises exponentially it is not clear that the energy will also be localised. Secondly, the particular form of the equations above means that previous existence proofs of breathers in coupled lattice ODEs do not hold here. For instance, the proof of the existence of breathers given in [11] requires linear coupling between adjacent pivots (see $\S 3$ for details) and that $A=B=0$ in the above set of ODEs. Similarly, the breather existence proofs in $[10,3]$ can cope with nonlinear coupling but require hardening inter-pivot potentials and

\footnotetext{
${ }^{\dagger}$ Centre for Nonlinear Mechanics, University of Bath, Bath, BA2 7AY, UK

${ }^{\ddagger}$ Department of Mathematical Sciences

$\S$ Department of Mechanical Engineering

ฯ Corresponding author; mascjb@bath.ac.uk
} 


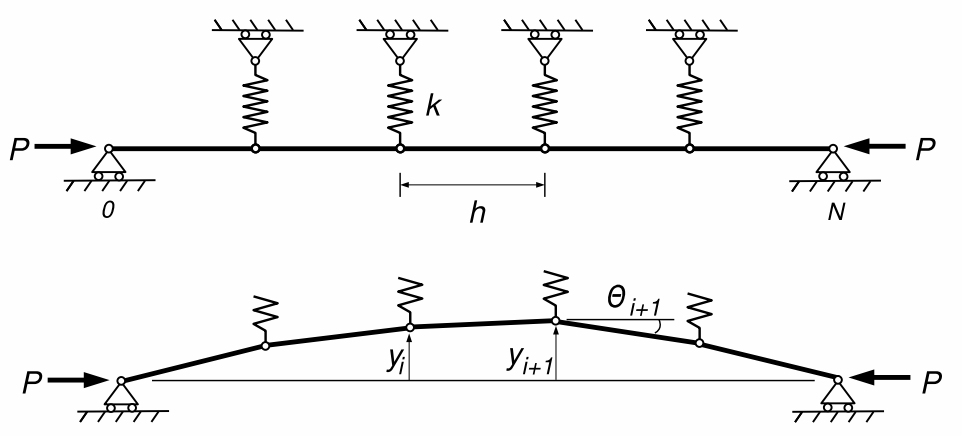

FIG. 1.1. The mechanical system in its flat state (top) and a displaced state (bottom) from [9] that will be studied in this article. It consists of a chain of freely pin jointed rigid links of length $h>0$ constrained to lie in a plane. The vertical springs are linear and the system is axially loaded by a force $P$ at the end points.

again $A=B=0$. In the mechanical system modelled in this paper the nonlinearities arise purely from the geometry of the mechanical system. For $p<0$ we see that the lattice exhibits behaviour characteristic of a hardening nonlinearity whilst for $p>0$ we see the characteristics of a softening nonlinearity.

In order to demonstrate the existence of breathers in this mechanical system we will use a combination of existing methods for locating breathers in nonlinear lattices, numerical continuation and direct simulation of the ODEs shown above. One method for locating breathers in nonlinear lattices is described by Marin and Aubry [12]. In this paper we use this method to find breathers in a simplified set of ODEs that are related to the ones shown above. We then use two stages of numerical continuation to locate breathers in the full set of ODEs. Some of the breathers found in the simplified set of ODEs do not survive the continuations into the fully nonlinear lattice and these branches end in either fold bifurcations or bifurcations with the linear modes of the lattice. This behaviour leads to numerical evidence that the fully nonlinear lattice is only able to support pivot centred breathers for even $N$ and link centred breathers for odd $N$.

Once we have located breathers in the mechanical lattice of Figure 1.1 we discuss their existence region in parameter space, their linear and nonlinear stability, and their energy localisation properties. We find that linearly stable sub-linear-spectrum simple breathers can be found for the lattice in compression $(p>0)$, tension $(p<0)$ and in the unloaded $(p=0)$ lattice also. The latter case is particularly surprising as in this case the only nonlinearity in the system is in the left hand side of the ODEs (1.1) shown above. The existence region in load-frequency space of these branches of simple breather solutions is bounded at higher frequencies by the lowest frequency linear mode of the system and at zero frequency as the breather solution approaches a heteroclinic connection between two static equilibrium states of the lattice.

Direct simulations of the mechanical lattice shown in Figure 1.1 show that the linearly stable, simple breathers are also stable to moderately large random perturbations. A breather with a random perturbation six percent the size of the breather's maximum amplitude shows no noticeable delocalisation after 1000 breather periods, whereas a linearly unstable breather solution has almost completely delocalised after just three breather periods. Finally, we see through direct simulation of the nonlinear 
lattice with random initial conditions and parameter values for which we know stable breather solutions can exist, that spontaneous energy localisation in this mechanical lattice is also possible. In the simulation performed here this manifested itself as what appears to be a spontaneously appearing moving breather in the lattice. This is similar to the moving discrete breathers reported in [13] and the spontaneous energy localisation seen in other nonlinear lattices by [6] and references therein.

The layout of this paper is as follows. In $\S 2$ we introduce a mathematical model of the mechanical system shown in Figure 1.1 and derive the ODEs (1.1) shown above. Section 3 explains the numerical methods and simplified lattices we use to find breather solutions in the full mechanical lattice of Figure 1.1. Some properties of these lattices are presented in $\S 4$. Section 5 shows examples of the implementation of the methods described in the previous two sections and locates breathers in the full mechanical lattice of Figure 1.1. Section 6 describes the parameter space existence region for these breather solutions and also discusses their energy localisation and nonlinear stability properties. In conclusion we summarise the results of the paper and present a preliminary numerical simulation that points towards the ability of energy to spontaneously localise in this mechanical lattice.

2. Modelling. The mechanical system we consider in this paper is the mechanical system studied in the time independent case by [9] and is shown in Figure 1.1. It consists of a freely pin jointed structure with $N$, mass-less, rigid links of length $h>0$. The displacements $y_{i}$, for $i=0, \ldots, N$, of the joints (mass $m$ ) are constrained to lie in the same plane and the system is constrained vertically at the ends so that $y_{0}=y_{N}=0$. The system is also subject to a steady axial load $P$. The shape of the mechanical system is determined entirely by the $N+1$ vertical pivot coordinates $y_{0}, \ldots, y_{N}$. Including the coordinate $\bar{x}$ to specify the horizontal coordinate of the lattice's centre of mass now completely specifies the state of the mechanical system. The aim of this section is to derive an ODE for the vertical displacements $y_{i}$ that models the fully nonlinear dynamic behaviour of this mechanical lattice. We do this by determining both the potential and kinetic energies of the system in terms of the $y_{i}$ coordinates which we then use to write down the Lagrangian subsequently derive the corresponding Euler-Lagrange equations.

We can write the potential energy of this system $V$ in terms of the energy stored in the springs $U$ and the work done by $P$ in producing a total end shortening $\mathcal{E}$, where

$$
\mathcal{E}=h \sum_{i=0}^{N-1}\left(1-\sqrt{1-\left(\frac{y_{i+1}-y_{i}}{h}\right)^{2}}\right) .
$$

After neglecting constant terms $V$ is written

$$
V=U-P \mathcal{E}=\frac{1}{2} k \sum_{i=0}^{N} y_{i}^{2}+P h \sum_{i=0}^{N-1} \sqrt{1-\left(\frac{y_{i+1}-y_{i}}{h}\right)^{2}} .
$$

We note here this model requires us to work with angular displacements of the lattice's links that satisfy $\Theta_{i} \in\left(-\frac{\pi}{2}, \frac{\pi}{2}\right)$ and so $\left|y_{i+1}-y_{i}\right|<h$. This means that we cannot consider situations where the links become vertical or where pivots cross over.

Writing down the kinetic energy in terms of the $y_{i}$ takes a little more work. We start by noticing that there are two straight-forward ways of writing the horizontal pivot displacements, $x_{i}$, in terms of the vertical displacements, $y_{i}$ : summing from the 
left of the lattice or summing from the right of the lattice.

$$
\begin{aligned}
\text { Left: } & x_{n}=x_{0}+h \sum_{i=1}^{n} \sqrt{1-\left(\frac{y_{i}-y_{i-1}}{h}\right)^{2}} \\
\text { Right: } & x_{n}=x_{N}-h \sum_{i=n+1}^{N} \sqrt{1-\left(\frac{y_{i}-y_{i-1}}{h}\right)^{2}} .
\end{aligned}
$$

Here we use the convention that sums for which the upper index is smaller than the lower index give the value zero. These two expressions seem slightly unsatisfactory as they give artificial significance to either the left, or right, end of the lattice. We can rectify this by averaging these to expressions to get a symmetric expression for the horizontal displacements $x_{i}$ in terms of the vertical displacements $y_{i}$ :

$$
x_{n}=\underbrace{\frac{1}{2}\left(x_{0}+x_{N}\right)}_{c}+\underbrace{\frac{h}{2}\left[\sum_{i=1}^{n} \sqrt{1-\left(\frac{y_{i}-y_{i-1}}{h}\right)^{2}}-\sum_{i=n+1}^{N} \sqrt{\left.1-\left(\frac{y_{i}-y_{i-1}}{h}\right)^{2}\right]}\right.}_{\mathcal{K}_{n}(\mathbf{y})} .
$$

The extra degree of freedom has been renamed $c$ and the combination of sums renamed $\mathcal{K}(\mathbf{y})$ to give the simpler expression

$$
x_{n}=c+\mathcal{K}_{n}(\mathbf{y}),
$$

which has time derivative

$$
\dot{x}_{n}=\mathcal{X}_{n}(\mathbf{y}, \dot{\mathbf{y}}, \dot{c})=\dot{c}+\frac{\mathrm{d} \mathcal{K}_{n}(\mathbf{y})}{\mathrm{d} y_{m}} \dot{y}_{m} .
$$

Here we have used the Einstein summation convention over repeated indices, $n, m=$ $0, \ldots, N$ and also $\mathbf{y}=\left(y_{0}, y_{1}, \ldots, y_{N}\right)^{\mathrm{T}}$. With this expression we can write down the Lagrangian for this mechanical system to give

$$
\mathcal{L}_{1}(\mathbf{y}, c, \dot{\mathbf{y}}, \dot{c})=\frac{m_{p}}{2} \dot{y}_{n} \dot{y}_{n}+\frac{m_{p}}{2} \mathcal{X}_{n}(\mathbf{y}, \dot{\mathbf{y}}, \dot{c}) \mathcal{X}_{n}(\mathbf{y}, \dot{\mathbf{y}}, \dot{c})-V(\mathbf{y}) .
$$

We now need to incorporate the vertically fixed end constraints $\left(y_{0}=y_{N}=0\right)$ into this model; we do this using two Lagrange multipliers $\mu_{1}$ and $\mu_{2}$ and forming the new Lagrangian function

$$
\mathcal{L}_{2}(\mathbf{y}, c, \dot{\mathbf{y}}, \dot{c})=\frac{m_{p}}{2} \dot{y}_{n} \dot{y}_{n}+\frac{m_{p}}{2} \mathcal{X}_{n}(\mathbf{y}, \dot{\mathbf{y}}, \dot{c}) \mathcal{X}_{n}(\mathbf{y}, \dot{\mathbf{y}}, \dot{c})-V(\mathbf{y})-\mu_{1} y_{0}-\mu_{2} y_{N} .
$$

The Euler-Lagrange equations for this Lagrangian can be found by evaluating

$$
\begin{aligned}
\frac{\mathrm{d}}{\mathrm{d} t}\left(\frac{\partial \mathcal{L}_{2}}{\partial \dot{y}_{k}}\right) & =\frac{\partial \mathcal{L}_{2}}{\partial y_{k}} \text { for } \quad k=0, \ldots, N \\
\frac{\mathrm{d}}{\mathrm{d} t}\left(\frac{\partial \mathcal{L}_{2}}{\partial \dot{c}}\right) & =\frac{\partial \mathcal{L}_{2}}{\partial c} .
\end{aligned}
$$

Evaluating (2.5) for the Lagrangian (2.4) gives

$$
\begin{aligned}
m_{p}\left(\delta_{k l}+\frac{\partial^{2} \mathcal{X}_{n}}{\partial \dot{y}_{l} \partial \dot{y}_{k}} \mathcal{X}_{n}+\frac{\partial \mathcal{X}_{n}}{\partial \dot{y}_{k}} \frac{\partial \mathcal{X}_{n}}{\partial \dot{y}_{l}}\right) \ddot{y}_{l}+m_{p}\left(\frac{\partial^{2} \mathcal{X}_{n}}{\partial y_{l} \partial \dot{y}_{k}} \mathcal{X}_{n}+\frac{\partial \mathcal{X}_{n}}{\partial \dot{y}_{k}} \frac{\partial \mathcal{X}_{n}}{\partial y_{l}}\right) \dot{y}_{l} \\
+m_{p}\left(\frac{\partial^{2} \mathcal{X}_{n}}{\partial \dot{c} \partial \dot{y}_{k}} \mathcal{X}_{n}+\frac{\partial \mathcal{X}_{n}}{\partial \dot{c}} \frac{\partial \mathcal{X}_{n}}{\partial \dot{y}_{k}}\right) \ddot{c}=-\frac{\mathrm{d} V}{\mathrm{~d} y_{k}}-\mu_{1} \delta_{0 k}-\mu_{2} \delta_{N k}
\end{aligned}
$$


for $k=0, \ldots, N$ whilst expression (2.6) becomes

$$
m_{p}\left(\frac{\partial \mathcal{X}_{n}}{\partial \dot{c}} \ddot{c}+\frac{\partial \mathcal{X}_{n}}{\partial y} \dot{y}+\frac{\partial \mathcal{X}_{n}}{\partial \dot{y}} \ddot{y}\right) \frac{\partial \mathcal{X}_{n}}{\partial \dot{c}}+m_{p}\left(\frac{\partial^{2} \mathcal{X}_{n}}{\partial \dot{c}^{2}} \ddot{c}+\frac{\partial^{2} \mathcal{X}_{n}}{\partial \dot{c} \partial y} \dot{y}+\frac{\partial^{2} \mathcal{X}_{n}}{\partial \dot{c} \partial \dot{y}} \ddot{y}\right) \mathcal{X}_{n}=0 .
$$

In these expressions we use the Einstein summation convection for repeated indices and $n, l, k=0, \ldots, N$. These differential equations should be solved along with the constraint equations $y_{0}=y_{N}=0$ making this a set of differential algebraic equations. However, we now show how we can eliminate the variable $c$ and convert this system in to a set of $N-1$ ordinary differential equations. We first eliminate $\ddot{c}$ by looking at equation (2.3) and noticing that for all $n, k$ and $l$

$$
\frac{\partial^{2} \mathcal{X}_{n}}{\partial \dot{y}_{k} \partial \dot{y}_{l}}=0, \quad \frac{\partial^{2} \mathcal{X}_{n}}{\partial \dot{c} \partial \dot{y}_{l}}=0, \quad \frac{\partial^{2} \mathcal{X}_{n}}{\partial \dot{c} \partial y_{l}}=0, \quad \frac{\partial^{2} \mathcal{X}_{n}}{\partial \dot{c}^{2}}=0, \quad \text { and } \quad \frac{\partial \mathcal{X}_{n}}{\partial \dot{c}}=1
$$

Inserting these simplifications into (2.8) leaves

$$
\begin{array}{r}
m_{p} \sum_{n=0}^{N}\left(\ddot{c}+\frac{\partial \mathcal{X}_{n}}{\partial y_{l}} \dot{y}_{l}+\frac{\partial \mathcal{X}_{n}}{\partial \dot{y}_{l}} \ddot{y}_{l}\right)=0 \\
\Rightarrow \ddot{c}=\frac{-1}{N+1} \sum_{n=0}^{N}\left(\frac{\partial \mathcal{X}_{n}}{\partial y_{l}} \dot{y}_{l}+\frac{\partial \mathcal{X}_{n}}{\partial \dot{y}_{l}} \ddot{y}_{l}\right) .
\end{array}
$$

To eliminate $\dot{c}$ we can find an expression for $\dot{c}$ in terms of $\mathbf{y}$ and $\dot{\mathbf{y}}$ by noticing that expression (2.9) is an exact derivative and so it implies

$$
\begin{aligned}
\frac{\mathrm{d}}{\mathrm{d} t}\left(m_{p} \sum_{n=0}^{N} \mathcal{X}_{n}(\mathbf{y}, \dot{\mathbf{y}}, \dot{c})\right) & =0 \\
m_{p} \sum_{n=0}^{N} \mathcal{X}_{n}(\mathbf{y}, \dot{\mathbf{y}}, \dot{c}) & =\beta,
\end{aligned}
$$

where $\beta$ is a constant. This expression is equivalent to saying that the centre of mass of the lattice, $\bar{x}$, travels at a constant velocity in the $x$ direction $^{1}, \dot{\bar{x}}=\beta$. If we constrain the system to have a horizontally fixed centre of mass we are able to set $\beta=0$. Using (2.3) in (2.11) then gives us

$$
\dot{c}=-\frac{1}{N+1} \sum_{n=0}^{N}\left(\frac{\partial \mathcal{K}_{n}(\mathbf{y})}{\partial y_{m}} \dot{y}_{m}\right) .
$$

Using this expression for $\dot{c}$ and (2.10) for $\ddot{c}$ we can eliminate $c$ from (2.7) to give

$$
\begin{aligned}
m_{p} \delta_{k l}+ & m_{p}\left(\frac{\partial \mathcal{X}_{n}}{\partial \dot{y}_{k}} \frac{\partial \mathcal{X}_{n}}{\partial \dot{y}_{l}}-\frac{1}{N+1}\left(\sum_{n=0}^{N} \frac{\partial \mathcal{X}_{n}}{\partial \dot{y}_{k}}\right)\left(\sum_{n=0}^{N} \frac{\partial \mathcal{X}_{n}}{\partial \dot{y}_{l}}\right)\right) \ddot{y}_{l} \\
+ & m_{p}\left(\frac{\partial^{2} \mathcal{X}_{n}}{\partial y_{l} \partial \dot{y}_{k}} \mathcal{X}_{n}+\frac{\partial \mathcal{X}_{n}}{\partial \dot{y}_{k}} \frac{\partial \mathcal{X}_{n}}{\partial y_{l}}-\frac{1}{N+1}\left(\sum_{n=0}^{N} \frac{\partial \mathcal{X}_{n}}{\partial \dot{y}_{k}}\right)\left(\sum_{n=0}^{N} \frac{\partial \mathcal{X}_{n}}{\partial y_{l}}\right)\right) \dot{y}_{l} \\
& =-\frac{\mathrm{d} V}{\mathrm{~d} y_{k}}-\mu_{1} \delta_{0 k}-\mu_{2} \delta_{N k} .
\end{aligned}
$$

\footnotetext{
${ }^{1}$ This is seen by differentiating the expression $\bar{x}=\frac{1}{N+1} \sum_{n=0}^{N} x_{n}$, twice with respect to time and using $\dot{x}_{n}=\chi_{n}(\mathbf{y}, \dot{\mathbf{y}}, c)$.
} 
Using (2.3) we can make this elimination of $c$ more explicit by replacing the function $\chi(\mathbf{y}, \dot{\mathbf{y}}, \dot{c})$ with $(2.3)$ and

$$
\frac{\partial \mathcal{X}_{n}}{\partial y_{l}}=\frac{\partial^{2} \mathcal{K}_{n}(\mathbf{y})}{\partial y_{m} \partial y_{l}} \dot{y}_{m}, \quad \frac{\partial \mathcal{X}_{n}}{\partial \dot{y}_{k}}=\frac{\partial \mathcal{K}_{n}(\mathbf{y})}{\partial y_{k}}, \quad \frac{\partial^{2} \mathcal{X}_{n}}{\partial \dot{y}_{l} \partial \dot{y}_{k}}=0, \quad \frac{\partial^{2} \mathcal{X}_{n}}{\partial \dot{y}_{k} \partial y_{l}}=\frac{\partial^{2} \mathcal{K}_{n}(\mathbf{y})}{\partial y_{k} \partial y_{l}}
$$

to leave

$$
\begin{aligned}
m_{p}\left(\delta_{k l}\right. & \left.+\frac{\partial \mathcal{K}_{n}}{\partial y_{k}} \frac{\partial \mathcal{K}_{n}}{\partial y_{l}}-\frac{1}{N+1}\left(\sum_{n=0}^{N} \frac{\partial \mathcal{K}_{n}}{\partial y_{k}}\right)\left(\sum_{n=0}^{N} \frac{\partial \mathcal{K}_{n}}{\partial y_{l}}\right)\right) \\
+m_{p}( & \frac{\partial^{2} \mathcal{K}_{n}}{\partial y_{k} \partial y_{l}}\left[\dot{c}(\mathbf{y}, \dot{\mathbf{y}})+\frac{\mathrm{d} \mathcal{K}_{n}}{\mathrm{~d} y_{m}} \dot{y}_{m}\right]+\frac{\partial \mathcal{K}_{n}}{\partial y_{k}} \frac{\partial^{2} \mathcal{K}_{n}}{\partial y_{m} \partial y_{l}} \dot{y}_{m} \\
& \left.-\frac{1}{N+1}\left(\sum_{n=0}^{N} \frac{\partial \mathcal{K}_{n}}{\partial y_{k}}\right)\left(\sum_{n=0}^{N} \frac{\partial^{2} \mathcal{K}_{n}}{\partial y_{l} \partial y_{m}} \dot{y}_{m}\right)\right)=-\frac{\mathrm{d} V}{\mathrm{~d} y_{k}}-\mu_{1} \delta_{0 k}-\mu_{2} \delta_{N k}
\end{aligned}
$$

for $k=0, \ldots, N$. Considering these differential equations with the constraint equations $y_{0}=y_{N}=0$ leads to a differential algebraic equation for the variables $y_{i}$ with $i=0, \ldots, N, \mu_{1}$ and $\mu_{2}$. We can almost trivially convert this into an ordinary differential equation by substituting $y_{0}=y_{N}=0$ into the above equations and then noticing that for $k=0$ and $k=N$ equation (2.13) can be solved to give explicit expressions for the Lagrange multipliers $\mu_{1}\left(y_{0}, \ldots, y_{N}\right)$ and $\mu_{2}\left(y_{0}, \ldots, y_{N}\right)$. We are then left with $N-1$ ODEs in the form of (2.13) for $k=1, \ldots, N-1$ with $y_{0}=y_{N}=0$. If we now let $\mathbf{y}=\left(y_{1}, \ldots, y_{N-1}\right)^{\mathrm{T}}$ we are able to define

$$
\begin{aligned}
{[A]_{k l}=} & \left(\frac{\partial \mathcal{K}_{n}}{\partial y_{k}} \frac{\partial \mathcal{K}_{n}}{\partial y_{l}}-\frac{1}{N+1}\left(\sum_{n=0}^{N} \frac{\partial \mathcal{K}_{n}}{\partial y_{k}}\right)\left(\sum_{n=0}^{N} \frac{\partial \mathcal{K}_{n}}{\partial y_{l}}\right)\right) \\
{[B]_{k l}=\left(\frac{\partial^{2} \mathcal{K}_{n}}{\partial y_{k} \partial y_{l}}\left[\dot{c}(\mathbf{y}, \dot{\mathbf{y}})+\frac{\mathrm{d} \mathcal{K}_{n}}{\mathrm{~d} y_{m}} \dot{y}_{m}\right]\right.} & +\frac{\partial \mathcal{K}_{n}}{\partial y_{k}} \frac{\partial^{2} \mathcal{K}_{n}}{\partial y_{m} \partial y_{l}} \dot{y}_{m} \\
& \left.-\frac{1}{N+1}\left(\sum_{n=0}^{N} \frac{\partial \mathcal{K}_{n}}{\partial y_{k}}\right)\left(\sum_{n=0}^{N} \frac{\partial^{2} \mathcal{K}_{n}}{\partial y_{l} \partial y_{m}} \dot{y}_{m}\right)\right)
\end{aligned}
$$

and

$$
[f(\mathbf{y})]_{k}=-\frac{\mathrm{d} V}{\mathrm{~d} y_{k}}
$$

for $k, l=1, \ldots, N-1, n, m=0, \ldots, N$ and the ODE modelling our mechanical system becomes

$$
m_{p}(I+A(\mathbf{y})) \ddot{\mathbf{y}}+m_{p} B(\mathbf{y}, \dot{\mathbf{y}}) \dot{\mathbf{y}}=f(\mathbf{y}),
$$

where $I$ is the $N-1$ by $N-1$ identity matrix.

It is convenient to non-dimensionalise the above equations using the scalings

$$
h \bar{y}_{i}=y_{i}, \quad h \bar{c}=c, \quad \bar{t} \sqrt{\frac{m}{k}}=t, \quad \frac{h k}{4} \bar{p}=p .
$$

These scalings leave one free parameter in this system: the nondimensionalised load $\bar{p}$. Dropping the bars from the nondimensional quantities we are left with the differential equations

$$
(I+A(\mathbf{y})) \ddot{\mathbf{y}}+B(\mathbf{y}, \dot{\mathbf{y}}) \dot{\mathbf{y}}=f(\mathbf{y})
$$


where $A, B, \mathcal{K}$ and $\dot{c}$ are given by the equations above with $h$ set to one and

$$
f(\mathbf{y})=\left(\begin{array}{c}
-y_{1}-\frac{p}{4} g\left(y_{2}-y_{1}\right)+\frac{p}{4} g\left(y_{1}\right) \\
\vdots \\
-y_{i}-\frac{p}{4} g\left(y_{i+1}-y_{i}\right)+\frac{p}{4} g\left(y_{i}-y_{i-1}\right) \\
\vdots \\
-y_{N-1}-\frac{p}{4} g\left(-y_{N-1}\right)+\frac{p}{4} g\left(y_{N-1}-y_{N-2}\right)
\end{array}\right)
$$

for $i=2, \ldots, N-2$ and $g(x)=x / \sqrt{1-x^{2}}$.

3. Method used to find breather solutions. As mentioned in the introduction, current existence proofs for breather behaviour in nonlinear lattices do not apply to lattice ODEs of the form (2.14). In addition to this there are also no direct numerical methods for finding breather solutions in these equations. This section describes how we located breather solutions in a simplified version of equations (2.14) before using numerical continuation to slowly increase the full nonlinearities these equations and hence find breathers in the mechanical lattice of Figure 1.1.

There are three aspects to our search for breather solutions. The first is the numerical method we have used to accurately find and continue time-periodic, timereversible solutions to these ODEs. The second is the numerical continuation procedure we have used to follow branches of breather solutions from the simplified lattice to the fully nonlinear mechanical lattice equations. The third aspect of the search for breather solutions is how we create breather solutions in the simplified mechanical lattice and which of the available solutions we choose to consider further. We now describe these methods.

Numerical methods. We are interested, in this paper, in time periodic solutions to multidimensional differential equations such as (2.14) and (3.5) (seen later). In order to find these computationally we first write the ODE system in the standard form $\dot{X}=F(X)$. Using the equations (2.14) as an example, we let $\mathbf{w}=\dot{\mathbf{y}}$,

$$
X=\left(\begin{array}{c}
\mathbf{y} \\
\mathbf{w}
\end{array}\right)
$$

and then equations (2.14) can be written in standard form thus

$$
\dot{X}=F(X) \equiv\left(\begin{array}{c}
\mathbf{w} \\
(I+A(\mathbf{y}))^{-1}(f(\mathbf{y})-B(\mathbf{y}, \mathbf{w}) \mathbf{w})
\end{array}\right) .
$$

This mechanical system is conservative, as are the multidimensional ODE systems we consider in this paper, and so we expect periodic solutions to exist in one parameter families which may be parametrised by the orbital period $T$. Also, when searching for periodic solutions we have to remove the time translation symmetry of a periodic solution and we do this by searching for time reversible solutions. We can confirm that the ODEs (2.14) are indeed reversible by defining the involution $\rho$ by

$$
\rho:\left(\begin{array}{c}
\mathbf{y} \\
\mathbf{w}
\end{array}\right) \rightarrow\left(\begin{array}{c}
\mathbf{y} \\
-\mathbf{w}
\end{array}\right)
$$

and using the expressions for $A(\mathbf{y})$ and $B(\mathbf{y}, \dot{\mathbf{y}})$ of the previous to show that

$$
\rho F(X)=-F(\rho X) .
$$


Similar calculations can verify this property for the other sets of ODEs seen later in this paper.

The search for time reversible periodic solutions rests on the following result.

Lemma 3.1. Let $X(t)$ be a solution to the dynamical system $\dot{X}=F(X)$ that for some $T>0$ satisfies $\rho X(0)=X(0)$ and $\rho X(T)=X(T)$. Also let $F$ be a reversible vector field, i.e. $\rho F(X)=-F(\rho X)$. Then there exists $\tau$ such that $X(t+\tau)=X(t)$, i.e. $X(t)$ is a time periodic solution.

Proof. See, for example, [8].]

After rescaling the time variable in (3.1) the above lemma allows us to see that the search for time reversible periodic solutions, with period $\tau$, is equivalent to finding solutions of the two point BVP

$$
\dot{X}=\tau F(X) \quad \text { with } \quad \mathbf{w}(0)=0 \quad \text { and } \quad \mathbf{w}(1)=0 .
$$

To find accurate solutions to this BVP we use a Newton based method (see [12] for further details). We search for zeros of the map $\mathcal{F}: \mathbb{R}^{N-1} \rightarrow \mathbb{R}^{N-1}$ which maps $\mathbf{y}(0)$ to $\mathbf{w}(T)$, where $T=2 \pi / \omega$ is the period of the periodic orbit sought and $\mathbf{y}, \mathbf{w} \in \mathbb{R}^{N-1}$. The numerical accuracy of this method depends on the Newton's method convergence criterion and the accuracy of the numerical integrator used to find $\mathbf{w}(T)$. In this work Matlab, with its IVP solver ode45 is used to compute individual time periodic solutions. Once we have a time periodic solution we use the numerical continuation software AUTO [4], which uses an adaptive collocation method to solve the relevant ODEs, to perform continuation of these periodic solutions and see how they change with the parameters of the system. Later, in $\S 6$ we see that in order to accurately determine the energy based properties of these periodic solutions numerically it is necessary to use one further numerical method that preserves the energy of the system better than AUTO and Matlab. The need for this method further discussed in $\S 6$ and the method itself is presented in Appendix A.

Continuation procedure. There are no direct methods for finding breather solutions in ODEs of the form (2.14) and so we first locate breathers in an artificially simplified version of the ODEs (2.14) using the method described in [12]. These artificially simplified ODEs are created by introducing continuation parameters $C$ and $\alpha$ such that when $C=0$ and $\alpha=0$ we can find breathers easily and when $C=1$ $\alpha=1$ we have the ODE system (2.14). We then numerically continue the breather solutions between these two parameter limits using the continuation code AUTO [4].

The first of the two parameters we introduce is $\alpha$ which enters the ODEs (2.14) in the following way:

$$
(I+\alpha A(\mathbf{y})) \ddot{\mathbf{y}}+\alpha B(\mathbf{y}, \dot{\mathbf{y}}, \dot{c}) \dot{\mathbf{y}}=f(\mathbf{y}) .
$$

When $\alpha=0$ the above equation becomes a set of nonlinearly coupled oscillators $\ddot{\mathbf{y}}=f(\mathbf{y})$, which then allows us to use the method of [12] to find breather solutions. This method requires the system $\ddot{\mathbf{y}}=f(\mathbf{y})$ to have a limit of one of its parameters (an anticontinuous limit) such that the system becomes a set of decoupled nonlinear oscillators. A trivial breather can be created in this limit: one oscillator oscillating while the others are stationary. If we write (3.2) for $\alpha=0$ explicitly we get

$$
\begin{aligned}
\ddot{y}_{1} & =-y_{1}-\frac{p}{4} g\left(y_{2}-y_{1}\right)+\frac{p}{4} g\left(y_{1}\right) \\
\ddot{y}_{i} & =-y_{i}-\frac{p}{4} g\left(y_{i+1}-y_{i}\right)+\frac{p}{4} g\left(y_{i}-y_{i-1}\right) \\
\ddot{y}_{N-1} & =-y_{N-1}-\frac{p}{4} g\left(-y_{N-1}\right)+\frac{p}{4} g\left(y_{N-1}-y_{N-2}\right),
\end{aligned}
$$


with $i=2, \ldots, N-1$ and $g(x)=x / \sqrt{1-x^{2}}$. We can see that there is no such nonlinear anticontinuous limit as the limit $p=0$ leads to decoupled linear oscillators. The technique used in [12] to get around the problem of the lack of such a limit is to introduce the change of variables given by

$$
z_{1}=y_{1}, \quad z_{i}=y_{i}-y_{i-1} \quad \text { for } \quad i=1, \ldots, N, \quad z_{N}=-y_{N-1} .
$$

Using this and subtracting (3.3) for $i=j+1$ from (3.3) for $i=j$ we get the following equations of motion for the variables $z_{j}$

$$
\begin{aligned}
\ddot{z}_{1} & =-z_{1}+\frac{p}{4} g\left(z_{1}\right)-\frac{p C}{4} g\left(z_{2}\right) \\
\ddot{z}_{j} & =-z_{j}+\frac{p}{2} g\left(z_{j}\right)-\frac{p C}{4}\left(g\left(z_{j+1}\right)+g\left(z_{j-1}\right)\right) \\
\ddot{z}_{N} & =-z_{N}+\frac{p}{4} g\left(z_{N}\right)-\frac{p C}{4} g\left(z_{N-1}\right) .
\end{aligned}
$$

for $j=2, \ldots, N-1$. We can now see where the second continuation parameter, $C$ is introduced. The parameter $C$ has been introduced so that when $C=0$ the system decouples into $N$ nonlinear oscillators. A trivial breather at frequency $\omega_{b}$ can be created in this limit: one oscillator oscillating while the others are stationary. As long as this breather is not at a frequency that is part of the linear spectrum of the lattice [11], it can then be numerically continued from this anticontinuous limit to the finite coupling regime to give the required breather. Once at $C=1$ we can recover the coordinates $y_{n}$ by using the expression

$$
y_{n}=\sum_{i=1}^{n} z_{i} \quad \text { for } \quad n=1, \ldots, N-1
$$

Once we have located a breather in equations (3.5) with $C=1$, or equivalently equations (3.2) with $\alpha=0$, we then perform another continuation, in $\alpha$ to find a breather in the fully nonlinear set of ODEs (2.14) and thus the mechanical lattice shown in Figure 1.1.

The answer to the question of which trivial breathers we can create in the anticontinuous limit, and whether these breathers can survive the continuations up to $C=1$ and $\alpha=1$, depends on the detailed properties of the nonlinear oscillators that result from setting $C=0$ in (3.5) and the linear spectrum of this lattice for $C>0$. This behaviour is investigated in the next section.

3.1. Anticontinuous limit. The previous two sections have described how we take easily created breather solutions from a simplified version of our mechanical lattice (the anticontinuous limit, $C=0$ in equations (3.5)) and then continue these breather solutions into the fully nonlinear lattice given by equations (2.14) (or (3.2) with $\alpha=1$ ). We now discuss these 'easily created' breathers in the anticontinuous limit.

The idea of the anticontinuous limit, as described in $[12,11]$ and others, is that creating a time periodic spatially localised oscillation in a set of uncoupled oscillators is easy, we set one oscillating whilst leaving the remainder stationary. We have restricted the search for breather solutions to time reversible solutions and so the two conditions we have on our 'trivial' breather solutions are that they are time periodic with frequency $\omega_{b}$ and that $\dot{z}(0)=0$ (where $z$ is the variable seen in equations (3.5)). 
To create a time reversible periodic solution to equations (3.5) with $C=0$ with frequency $\omega_{b}$, we set each individual oscillator oscillating with a frequency $\omega_{z_{i}}$ such that $n \omega_{z_{i}} / 2=\omega_{b}$ for any $n \in \mathbb{N}$. We ensure that this solution is time reversible by choosing the initial conditions $z_{i}(0)=a_{i} z_{i}^{\max }$ for each $i$ where $a_{i} \in\{-1,0,1\}$ and $z_{i}^{\max }>0$ is chosen so that oscillator $i$ is oscillating at frequency $\omega_{z_{i}}$. This method leads to many different types of solution, some of which are not spatially localised (these are often referred to as multibreathers). Since, in this paper, we are interested in localisation in this mechanical lattice we shall restrict our attention to two of the very simplest type of localised solutions which we will call the pivot centred breather $(P C)$ the link centred breather $(L C)$. These breathers are characterised by the amplitudes of the nonlinear oscillators at $t=0$ in the anticontinuous limit.

$$
L C: \quad z_{i}=\left\{\begin{array}{ll}
z\left(\omega_{b}\right) & i=i^{*} \\
z_{i}=0 & \text { otherwise }
\end{array}, \quad P C: \quad z_{i}= \begin{cases}z\left(\omega_{b}\right) & i=i^{*} \\
-z\left(\omega_{b}\right) & i=i^{*}+1 \\
z_{i}=0 & \text { otherwise }\end{cases}\right.
$$

where $i^{*}=\lfloor(N+1) / 2\rfloor$. We note here that we have used the terminology link and pivot centred to refer to the original mechanical lattice (of Figure 1.1) rather than the transformed, anticontinuous limit lattice. This means that in the coordinates $z_{i}$ for $i=1, \ldots, N$ as shown above these labels appear to be the wrong way around. Transforming back to the original lattice coordinates removes this issue.

The decoupled oscillators that arise from setting $C=0$ in equation (3.5) cannot oscillate at any frequency. The attainable set of frequencies is determined by the behaviour of these decoupled nonlinear oscillators. Similarly, not all breathers created in this anticontinuous limit will be able to be continued into the fully nonlinear mechanical lattice regime. This behaviour is mostly determined by the linear spectrum of the ODEs seen above. The next section determines the behaviour of the decoupled nonlinear oscillators and the linear spectra of the lattices shown above.

4. Lattice properties. In order to start our detailed search for breathers in the nonlinear lattices presented in the previous section we need to know some of their detailed properties. Firstly, in order to choose a suitable frequency of oscillation in the anticontinuous limit lattice (ODEs (3.5) with $C=0$ ) we need to know the amplitude frequency dependence of these decoupled nonlinear oscillators. This is determined in the following section. It is also known that the linear spectrum of a lattice plays an important role in the existence theory for breathers as it is shown in [11] that we cannot continue a breather from the anticontinuous limit if the breather is at a frequency that resonates with the linear spectrum. This means that we require $n \omega_{b} \neq \omega_{i}$ for all $n \in \mathbb{N}$ and $\omega_{i} \in \sigma$ where $\omega_{b}$ is the breather frequency and the set $\sigma$ is the linear spectrum of the lattice. Thus we look at these linear spectra in $\S 4.2$ below.

4.1. Anticontinuous limit: oscillators. Setting $C=0$ in (3.5a) and (3.5c) leads to two nonlinear oscillators of the form

$$
\ddot{z}_{n}=-z_{n}+\frac{p}{4} \bar{g}\left(z_{n}\right)
$$

while $C=0$ in (3.5b) leads to $N-2$ nonlinear oscillators of the form

$$
\ddot{z}_{n}=-z_{n}+\frac{p}{2} \bar{g}\left(z_{n}\right) .
$$

The first oscillators (4.1) are different from the oscillators (4.2) due to boundary effects. In this paper we wish to consider breather solutions for which the vertical 

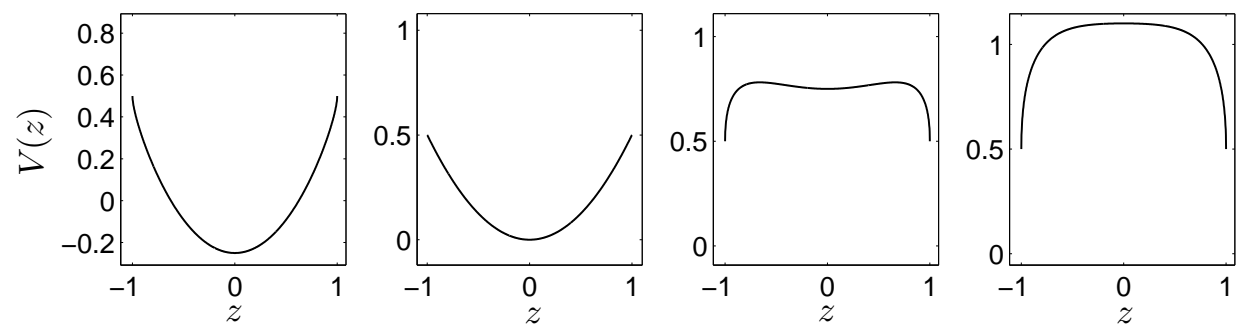

FIG. 4.1. Plots of the potential function (4.3) for the $p$ values, from left to right, $p=$ $-0.5,0,1.5,2.2$. This illustrates the behaviour of the interior nonlinear oscillators obtained from the anticontinuous $(C=0)$ limit of the lattice ODEs (3.3), as described in the text.

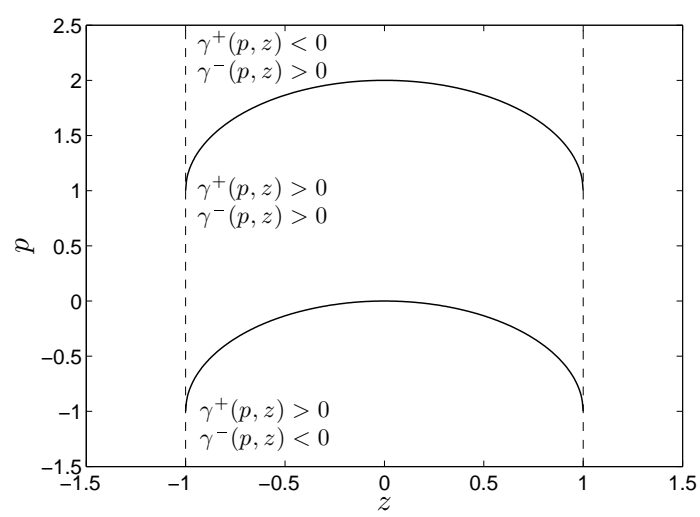

Fig. 4.2. For the expression (4.4) to be valid we require $\beta^{2}>0$. If we define $\gamma^{+}(p, z)=2 V(z)+p$ and $\gamma^{-}(p, z)=2 V(z)-p$ then $\beta^{2}>0$ when $\gamma^{+}$and $\gamma^{-}$are greater than zero. This occurs for the central region shown in this figure. The vertical dotted lines show the maximum and minimum possible values of $z, z= \pm 1$.

displacement of the lattice is localised to some interior points of the lattice and so we will not consider, in detail, the behaviour of the oscillators (4.1). The oscillators (4.2) are Hamiltonian with Hamiltonian function

$$
H=\frac{z^{2}}{2}+V(z), \quad \text { and } \quad V(z)=\frac{z^{2}}{2}+\frac{p}{2} \sqrt{1-z^{2}} .
$$

The qualitative behaviour of this Hamiltonian oscillator for different values of $p$ can be found by considering the behaviour of the function $V(z)$, which is plotted for the $p$ values $p=-0.5,0,1.5,2.2$ in Figure 4.1. This allows us to get our first insight into the physical behaviour of this system. The plots in Figure 4.1 show us that putting the lattice under tension $(p<0)$ tends to make the system behave as if the nonlinearity is a hardening nonlinearity and the frequency of oscillation increases with amplitude. However, with the lattice under compression $p>0$ the converse is true the system's nonlinearity appears to be of softening type. This means that, at least initially, we expect to look for breathers at frequencies above the linear spectrum for $p<0$ and breathers below the linear spectrum for $p>0$. This oscillator behaviour is summarised in the following table. 


\begin{tabular}{llll}
$p$ & Oscillatory domain & $\omega_{\max }$ and $\omega_{\min }$ & Characteristic \\
\hline$p<0$ & $|z|<1$ & $\omega_{\min }=\omega_{\text {lin }}, \omega_{\max }=\omega(1)$ & Hardening \\
$p=0$ & $z \in \mathbb{R}$ & $\omega_{\min }=\omega_{\max }=\omega_{\text {lin }}$ & Linear \\
$0<p<2$ & $|z|<\sqrt{1-p^{2} / 4}$ & $\omega_{\min }=0, \quad \omega_{\max }=\omega_{\text {lin }}$ & Softening
\end{tabular}

The frequency of small oscillations for the above nonlinear oscillator (see equation $(4.2))$ is

$$
\omega_{l i n}=\sqrt{1-\frac{p}{2}}
$$

For the $p$ values and oscillation amplitudes, $z$, shown in Figure 4.2 we can determine the oscillation frequency analytically and this is given by

$$
\omega(z)=\left(\frac{4 a}{\pi \sqrt{2 V(z)-p}}\left[2 \Pi\left(-a^{2} ; \phi(z) \mid \beta^{2}\right)-F\left(\phi(z), \beta^{2}\right)\right]\right)^{-1}
$$

where

$$
\begin{array}{r}
a^{2}=\frac{4-4 h-\sqrt{\left.(4 h-4)^{2}-4(4 h+p)(4 h-p)\right)}}{4 h-2 p}, \quad \beta^{2}=\frac{(2 h+p) a^{4}}{2 h-p} \\
h=V(z), \quad \phi(z)=\sin ^{-1}\left(\frac{1}{a} \tan \left(\frac{1}{2} \sin ^{-1}(z)\right)\right),
\end{array}
$$

and $F$ and $\Pi$ are the incomplete elliptic integrals of the first and thrid kinds respectively. Using expression (4.4) we can determine the maximum and minimum oscillation frequencies that the nonlinear oscillators defined by (4.3) can achieve. The dependence of these frequencies on $p$ is plotted in Figure 4.3 as solid lines. This information will be used for comparison with the linear spectrum which is found in the next section.

4.2. Linear Spectra. We have two lattice equations we are considering in this paper, (3.5) and (3.2). These are the two lattices we have to perform continuation on to reach the full mechanical lattice. In the first of these lattices, (3.5), the continuation parameter $C$ does affect the linear behaviour. The linear spectrum of this lattice is derived from the set of eigenvalues, $\left\{\lambda_{k}\right\}$, of the matrix $\mathcal{A} \in \mathbb{R}^{N \times N}$, with

$$
\mathcal{A}=\left(\begin{array}{cccccc}
\frac{p}{4}-1 & -\frac{p C}{4} & & & & \\
-\frac{p}{C} 4 & \frac{p}{2}-1 & -\frac{p C}{4} & & & \\
& \ddots & \ddots & & & \\
& & & -\frac{p}{C} 4 & \frac{p}{2}-1 & -\frac{p C}{4} \\
& & & & -\frac{p}{C} 4 & \frac{p}{4}-1
\end{array}\right)
$$

through the relation $\omega_{k}=\sqrt{-\lambda_{k}}$ for $k=1, \ldots, N$. The variation of $\omega_{k}$ with $C$ is shown in Figure 4.4 for $p=-0.5$ and $p=-0.5$ in (a) and (c) respectively. Parts (b) and (d) of Figure 4.4 show the corresponding eigenvectors and show that for $p<0$ the highest frequency modes have the shortest spatial wavelength whilst for $p>0$ the highest frequency modes have the longest spatial wavelength. We can also see that, as expected, at $C=0$ the system decouples into $N-2$ oscillators with frequency $\sqrt{1-p / 2}$ and the two oscillators at the boundaries with frequency $\sqrt{1-p / 4}$. In 


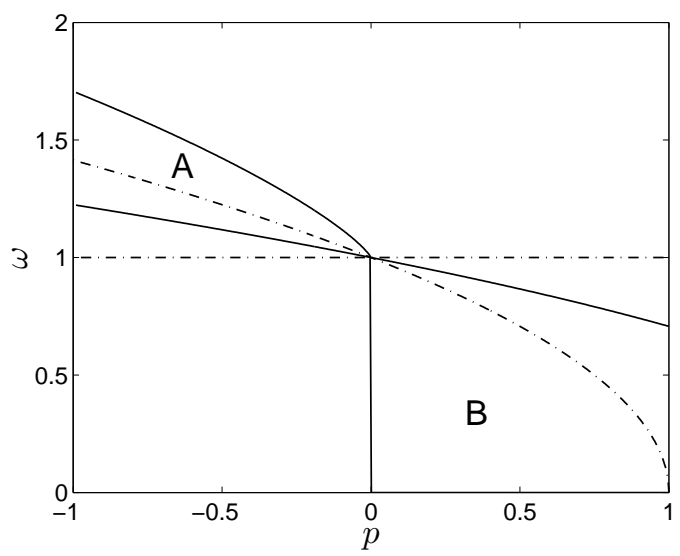

FIG. 4.3. This shows the maximum and minimum frequencies, $\omega_{\max }$ and $\omega_{\min }$, achievable in the nonlinear oscillators (4.2) for $-1<p<1$ as solid lines. The dashed lines show the lowest and highest frequency linear modes in the nonlinear lattice (3.5) for $C=1$. Regions $A$ and $B$ show the parameter regions for which we expect branches of simple breathers to exist between $C=0$ and $C=1$.

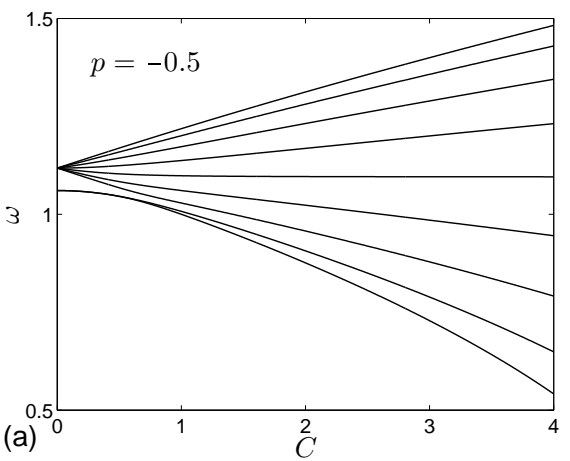

$z_{i}(0)$

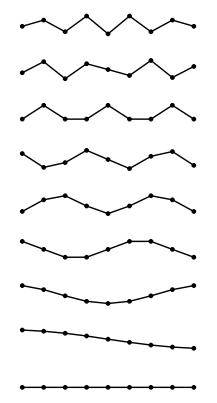

(b)
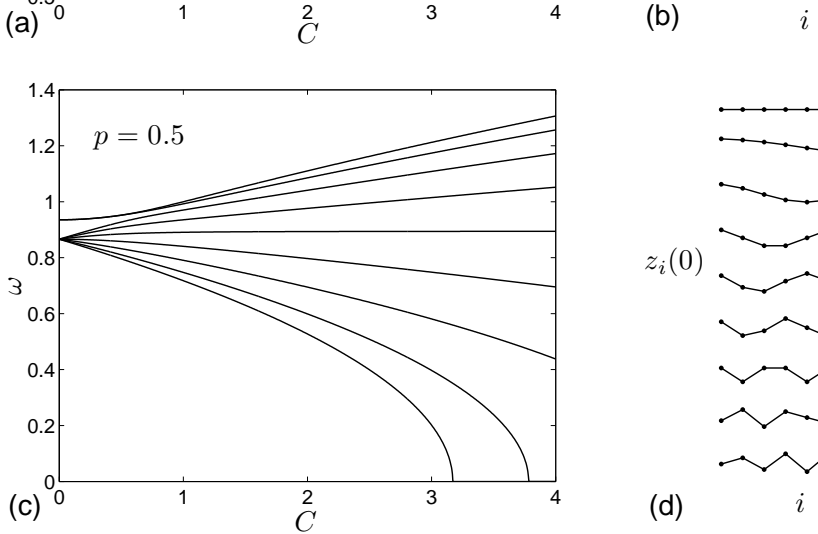

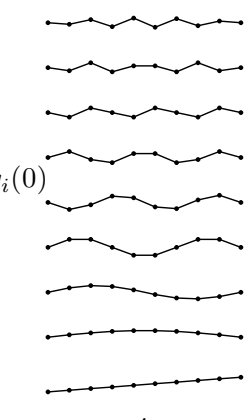

$i$

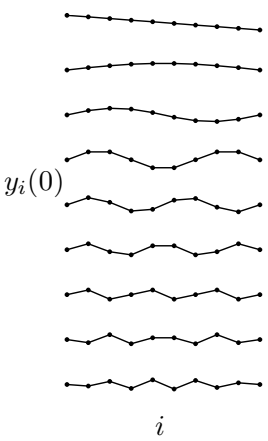

FIG. 4.4. The two plots on the left show how the frequencies in the linear spectrum of lattice (3.3) change as the parameter $C$ is increased from the anticontinuous limit for $N=9$ and $p=-0.5$ (top) and $p=0.5$ (bottom). The plots on the right show the linear mode shapes at $C=1$ in the $z$ coordinates and transformed into the $y$ coordinates using (3.6). We can see that for $p>0$ the highest temporal frequency mode has the longest spatial wavelength, and for $p<0$ the situation is reversed. 
order to locate a breather in the fully nonlinear mechanical lattice we have to continue the trivial breather solution from the anticontinuous limit $(C=0)$ of equation (3.5) up to $C=1$. For this to be possible the frequency of the breather must not collide with the linear spectrum shown in Figure 4.4. To see the parameter values that this condition allows the maximum and minimum linear frequencies at $C=1$ are shown in Figure 4.3 as dashed lines. This then tells us that the two parameter regions in which we are able to search for breathers are the regions denoted A and B in Figure 4.3 .

In the second lattice we shall perform continuation on, (3.2), the continuation parameter only affects the nonlinear terms and so the linear spectrum for this lattice is the same as for the lattice above with $C=1$. It turns out that when $C=1$ this linear spectrum can be determined analytically to give

$$
\omega_{k}^{2}=1+\frac{p}{2}\left(\cos \frac{k \pi}{N}-1\right) .
$$

5. Results: computations of breather solutions. We now have all the information needed to present the numerical results of our search for breather solutions in the mechanical lattice shown in Figure 1.1.

5.1. Continuation one. In this section we consider the behaviour of the branches of link and pivot centred breathers found in the anticontinuous limit in $\S 3$ as we increase the parameter $C$ from 0 . There are two bifurcation scenarios for the branches of breather solutions continued away from the anticontinuous limit; these are illustrated in Figure 5.1. The first bifurcation scenario, (a) of Figure 5.1, is that the breather frequency encounters the edge of the linear spectrum of the lattice as $C$ is increased, the breather delocalises and the branch disappears in a bifurcation with the band edge linear mode. As long as this bifurcation with the linear mode happens for $C>1$ we can still locate a breather at $C=1$ as is shown in (b) of Figure 5.1. The second type of breather branch behaviour as we increase $C,(\mathrm{c})$ of Figure 5.1, is that the breather branch disappears in a fold bifurcation with another, related breather branch. Again, as long as this fold bifurcation occurs at $C>1$ we can locate breather solutions at $C=1$ (plots (d) and (e)) as required for the second stage of numerical continuation.

Predicting which values of $N, p$ and $\omega$ lead to each of these two different situations is difficult. Similarly, determining which branches will reach $C=1$ and which will not is also not clear. Having said this, it appears to be the case that for the majority of parameter values, $p$ and $\omega$, when $N$ is odd the branch of link centred breathers tends to terminate in a bifurcation with the band edge linear mode and when $N$ is even this branch tends to terminate in a fold bifurcation. The converse is the case when considering the pivot centred breathers. The branches of pivot centred breathers tend to terminate in a bifurcation with the band edge linear mode for $N$ even and in fold bifurcation when $N$ is odd.

5.1.1. Breathers for values of $\omega$ above the linear spectrum. The discussion so far applies to locating breathers at frequencies below the linear spectrum. We can see from Figure 4.3 that it should be possible, using the same methods as in the previous section, to determine breathers above the linear spectrum for $p<0$ by choosing frequencies in region A. Frequencies in this region are achievable in the anticontinuous limit of lattice (3.5) and will not collide with the highest frequency linear mode for $C \leq 1$. Attempting to perform this continuation from $C=0$ to $C=1$ leads to a problem with the particular model chosen in $\S 2$. As we continue this branch 

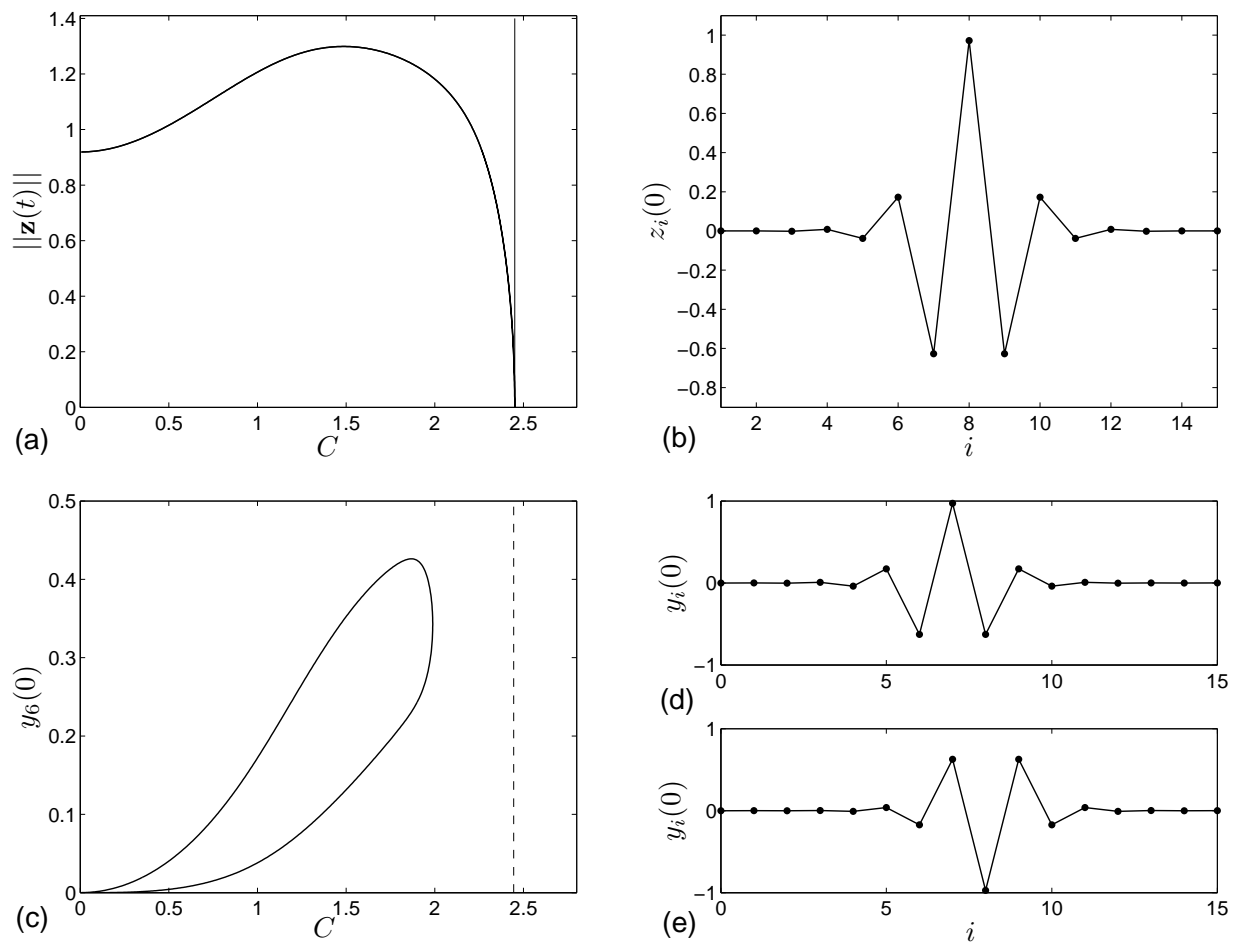

(d)

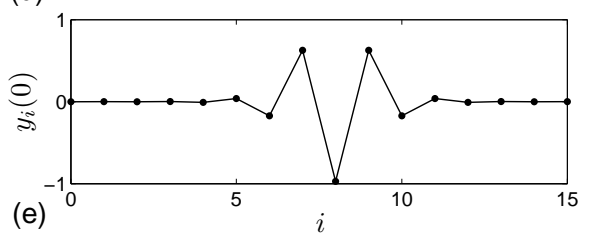

FIG. 5.1. Examples of continuations from the anticontinuous $(C=0)$ limit of lattice ODEs (3.5) for $p=0.3$ and $\omega=0.7$. Plots $(a)$ and (b) are for $N=15$ and plots $(c)-(e)$ are for $N=16$. Plot (a) shows how this breather reaches $C=1$ and then at a higher $C$ value collides with the lowest frequency linear mode at the $C$ value marked by a vertical black line. Plot (b) shows the breather profile at $C=1$. Plot (c) shows how for even $N$ the simple breather branch disappears in a fold bifurcation before reaching the edge of the linear spectrum indicated by the vertical dashed line. Plots (d) and (e) show the breather profile at $C=1$ for the upper and lower branches respectively shown in $(c)$.

from $C=0$ it the breather solutions appear to move into the region where $\left|z_{i}\right|>1$ for some $i$. Equivalently, one of the mechanical links is trying to attain an angle with the horizontal of greater than $\pi / 2$ for $C \ll 1$. To consider these breathers will require a more general model than that used here and so we postpone investigation of these breathers above the linear spectrum until further work has been completed.

5.2. Continuation two. In the previous section we saw that it is possible to find breather solutions in the intermediate lattice equations, (3.5), at $C=1$. Via the coordinate transformation (3.4) this is equivalent to finding a breather solution in the lattice equations (3.2) with $\alpha=0$. We now consider the bifurcation behaviour of these breather branches as we increase $\alpha$ from zero to one.

In this continuation the distinction between the bond centred and site centred breathers is more clear. Again, there appear to be two fates for the breather branches as we continue $\alpha$ up from zero (see Figure 5.2). Either the branch disappears in a fold bifurcation for a small value of $\alpha$ (Figure $5.2(\mathrm{~d})$ ) or the branch continues towards higher and higher values of $\alpha$ with the breather amplitude asymptoting to zero as $\alpha$ increases (Figure $5.2(\mathrm{a})$ ). The first of these situations occurs for the bond centred breathers when $N$ is even and for the site centred breathers when $N$ is odd. The 
(a)
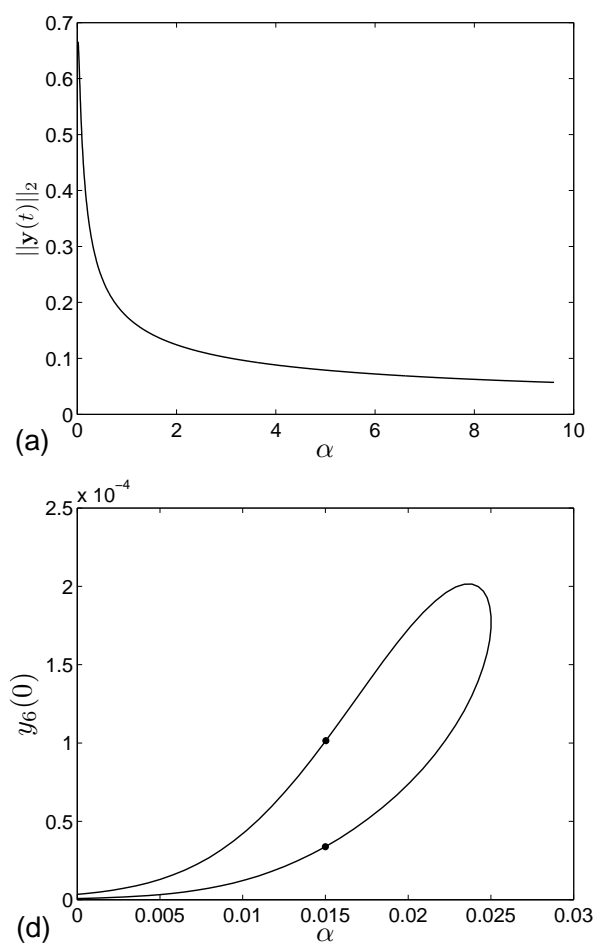
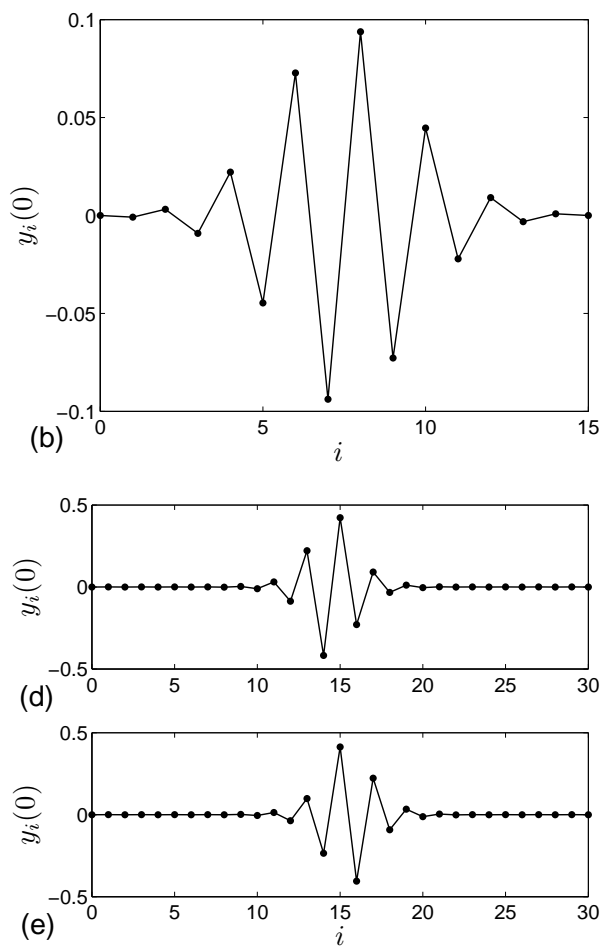

FIG. 5.2. Examples of the two different types of behaviour when following a branch of link centred breather solutions towards higher $\alpha$ values. Pane (a) shows an example of a branch that appears to extend to infinite values of $\alpha$ with an ever decreasing breather amplitude (parameters $N=15, p=0.3, \omega=0.7$ ). Pane (b) shows the breather at $\alpha=1$ on the branch in pane (a). Pane (c) shows an example of a branch of breather solutions that is destroyed in a fold bifurcation. The parameters here are $N=30, p=0.3, w=0.7$. The solutions marked by $\bullet$ in (c) are shown in (d) and (e), with solution (d) being the higher dot.

second situation occurs for the bond centred breathers when $N$ is odd and for the site centred breathers when $N$ is even.

Only the first of these two bifurcation scenarios leads to finding a breather in the full mechanical lattice. An example of such a breather is shown in Figure 5.2 (b). Now that we have located breathers in the mechanical lattice of Figure 1.1 we now go on to investigate some of their properties.

6. Breather Properties. We saw in the previous section that it is possible to find breathers in the fully nonlinear mechanical lattice described by the ODE system (2.14). The purpose of this section is to describe some of their properties and to investigate whether these breathers have similar properties to breathers found in simpler multidimensional ODEs in the literature.

6.1. Existence and Stability. Now that we have located both a breather in the mechanical lattice the next important questions are for what parameters in load frequency space do these breathers persist and are they linearly (and nonlinearly) stable. These questions are particularly important with regard to the possibility of observing these solutions experimentally: there is very little hope if they only exist for very specific parameter values or are dynamically unstable. 

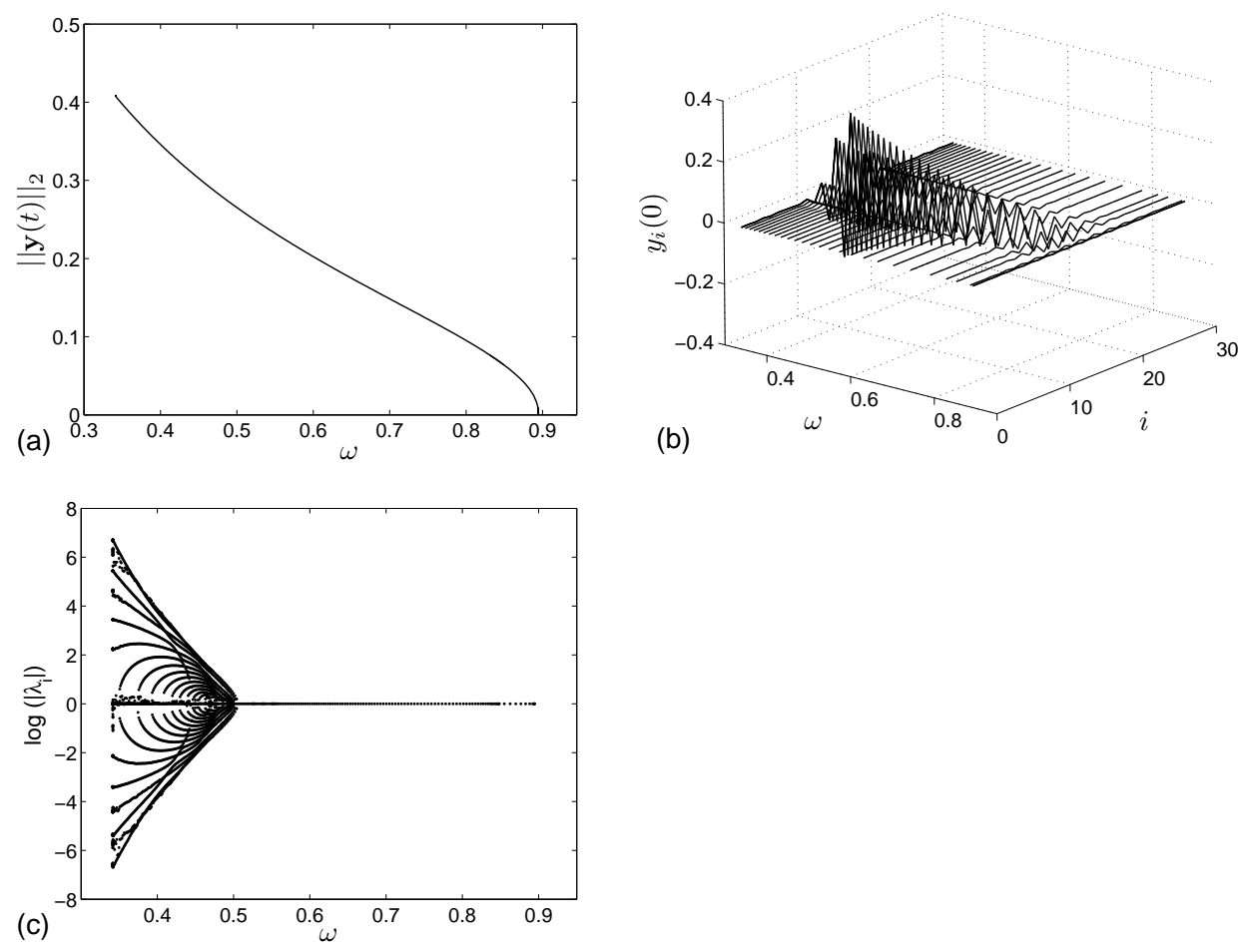

FIG. 6.1. This shows the behaviour of a link centred breather branch for $N=29$ and $p=0.2$ as it is continued in the breather frequency $\omega$. As $\omega$ approaches zero the breather amplitude increases ((a) and (b)) and the periodic solution approaches a heteroclinic connection between two static equilibrium states of the lattice. At the other end of the branch the branch bifurcates with the band edge linear mode and the breather delocalises. Pane (c) shows the absolute values of the Floquet multipliers for this breather solution and we see the breather looses its linear stability at about $\omega=0.5$.

Figure 6.1 summarises the results of a numerical continuation that shows how a link centred breather changes shape and stability as we change the frequency of the breather solution. As the breather frequency is increased, since this breather exists at frequencies below the linear spectrum of the lattice, the breather frequency gets closer and closer to the linear mode at the lower spectrum edge. Eventually the breather delocalises and bifurcates with this linear mode (Figure 6.1 (a) and (b)), which is behaviour seen in many other systems which can support discrete breathers. As we reduce the breather frequency, the breather solutions on this branch approach a heteroclinic connection between two static equilibrium states of the mechanical lattice. As we get close to this heteroclinic connection it becomes increasingly difficult to follow the branch numerically and so the plots in Figure 6.1 stop at $\omega \approx 0.35$.

Plot (c) of Figure 6.1 shows us the absolute values of the Floquet multipliers of this time periodic breather solution. This shows that at around $\omega=0.5$ this breather branch looses its linear stability and becomes more and more unstable as the breather frequency approaches zero. On this Figure we finally note that this breather branch is at constant load and the changing breather frequency has little affect on the localisation width. This figure, therefore, gives us the frequency-amplitude relation for this discrete breather. 

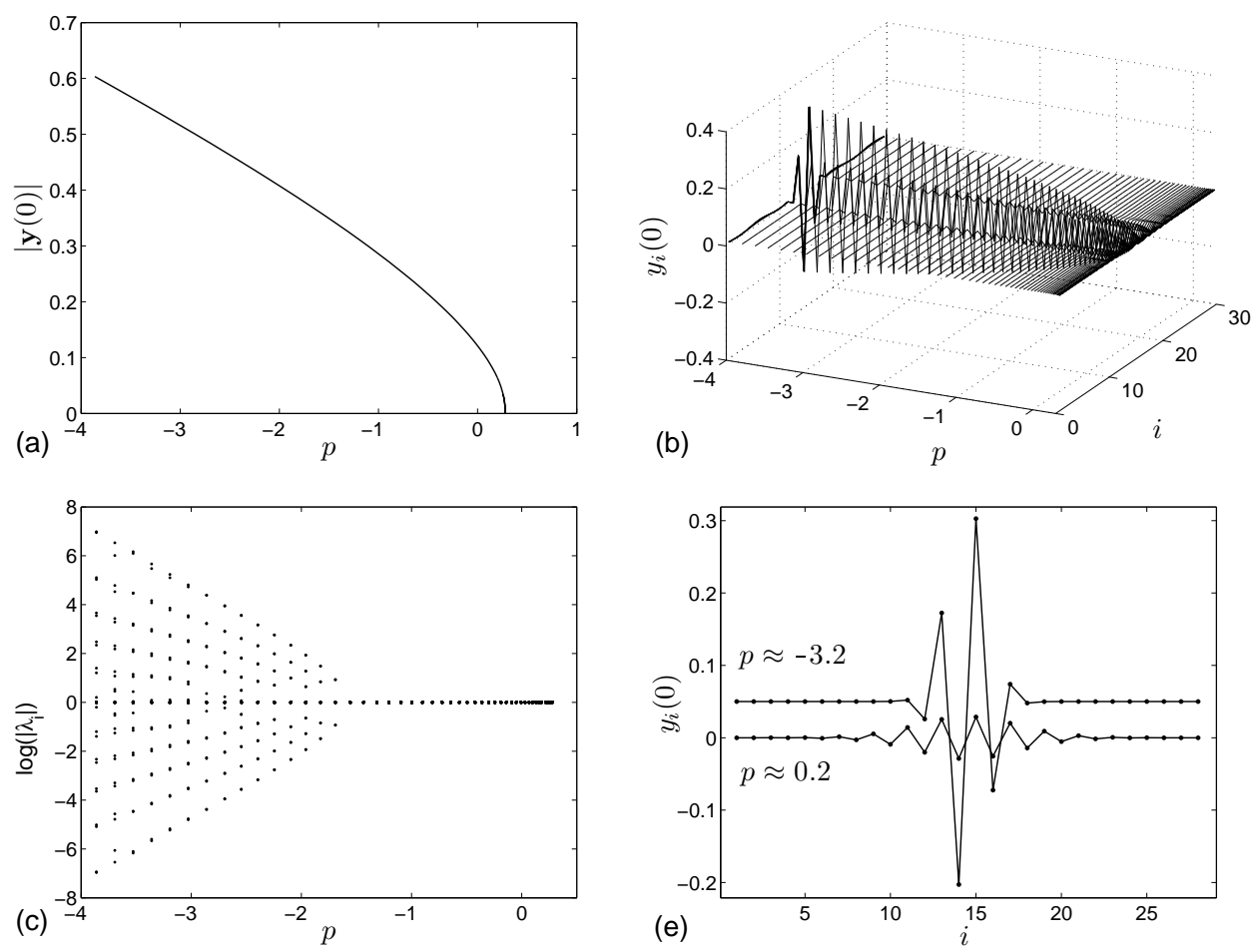

FIG. 6.2. Plots (a) and (b) show how the amplitude of a link centered breather solution, with $N=29$ and $\omega \approx 0.84$, increases as the load $p$ on the lattice is reduced. Plot (c) shows that absolute values of the Floquet multipliers for this breather solution and we see that the lienar stability is load at $p \approx=-1.6$. Plot (d) shows how reducing $p$ affects the localisation width of the breather. The plot for $\approx-3.2$ has been displaced upwards on the plot by 0.05 to make comparing the two breather shapes easier.

On the other hand, Figure 6.2 shows how the same link centred breather solution, with $N=29$, changes as we alter the axial load on the lattice. As $p$ is increased, as with $\omega$ described above, we eventually reach the edge of the linear spectrum of the lattice and the branch of breather solutions disappears in a bifurcation with this band edge linear mode. As $p$ is reduced, however, we see some different behaviour. As can be seen in Figure 6.2 (b) and (d) reducing the load $p$ increases the amplitude and reduces the localisation width so that for $p \approx-3.2$ in (d) we have a sharply localised breather with a relatively large amplitude. Pane (c) shows, again, that the size of the region of linear stability is significant with this breather branch loosing its linear stability at around $p=-1.6$.

The slightly surprising aspect of the results presented in Figure 6.2 is that the branch of breather solutions shown there passes continuously through the change of load on the lattice from a compressive situation $(p>0)$ through the unloaded state, into a situation where the lattice is under tension. We can see through equations (2.14) and (2.15) (p6) that when $p=0$ the only nonlinearity in the equations of motion for this system is in the mass matrix terms on the right hand side of (2.14). This contrasts with most other ODE systems supporting breather solutions which contain some type of nonlinearity in the potential, or left-hand-side of (2.14). 


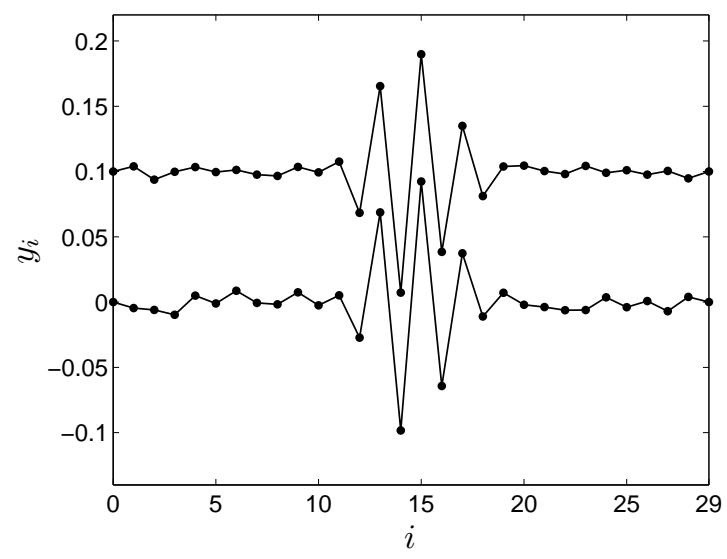

FIG. 6.3. This shows a perturbed breather profile $(N=29 p \approx-0.2432$ and $\omega \approx 0.8497)$ at $t=0$ (lower) and $t=1000 \tau_{b}$ (upper) where $\tau_{b}$ is the breather period. This demonstrates that this breather solution as well as being linearly stable is also stable to moderately large random perturbations.

6.1.1. Breather nonlinear stability. We have seen in the previous section that simple breather solutions can be linearly stable in this nonlinear mechanical lattice. In order to observe them experimentally it is useful to know their lifetime away from the exact solution's trajectory. In order to investigate this we have directly simulated ${ }^{2}$ the ODEs (2.14) using the solutions found in the previous section (labelled $y^{*}$ below) with a small random perturbation added to each of the pivot displacements. This perturbation took the form

$$
y_{i}(0)=y_{i}^{*}(0)+\Delta y \quad \text { with } \Delta y \sim U(-\epsilon, \epsilon)
$$

with $\epsilon=1 \times 10^{-2}$.

The result of this simulation for the linearly stable, link centred, simple breather solution with $N=29, p \approx-0.2432$ and $\omega \approx 0.8497$ is shown in Figure 6.3. With a perturbation to the breather's initial conditions as above the amplitude of the perturbation is around six percent of the breather's maximum vertical displacement and even after 100 breather periods we can see that the breather solution has not noticeably delocalised. In contrast, performing the same simulation on a linearly unstable breather solution leads to total delocalisation of the breather solution in a time less than three breather periods.

6.2. Localisation properties. It was mentioned in $\S 1$, and can be seen by considering the mechanical system in Figure 1.1, that it is not clear that if the vertical displacement of this mechanical system localises that the energy does also. This question arises because the vertical displacement of the central pivots affects the horizontal displacement of the pivots in the tails. This means that we need to look more closely at the kinetic energy present in the breather tails and see whether this is significant.

The adaptive collocation method used by AUTO to solve the differential equations when continuing these periodic solutions is used because it is quick, accurate and efficient for numerical continuation. However, it does not preserve the total energy

\footnotetext{
${ }^{2}$ These simulations were performed using the numerical method presented in the Appendix A
} 
well in Hamiltonian dynamical systems such as the mechanical lattice we are looking at. In order to compare the energy in the core and the tails for these breathers we have thus used a different method to solve for the time dependence of the mechanical system shown in Figure 1.1. This is a constraint based method solved numerically with a generalised RATTLE algorithm (see [8, §VI.1.3]) which is explained further in Appendix A. This numerical method keeps the total energy in the system constant to a much better accuracy that AUTO's numerical integrator. The initial conditions for this numerical integration are taken from the boundary value solution generated by AUTO. This change of numerical integrator leads to a slight discrepancy between the exact trajectories of these two methods as can be seen by noticing that in Figure 6.4 the oscillations do not have period one exactly.

Most breather solutions in the literature show exponentially localised displacement in the spatial direction. We can see from Figure 6.5 (b) that the vertical displacement of these mechanical breathers also appears to be, at least, exponentially localised. Now to consider the energy behaviour.

The mechanical system shown in Figure 1.1 is Hamiltonian and if we give pivot $i$ the horizontal coordinate $x_{i}$ and vertical coordinate $y_{i}$ we can write the Hamiltonian function as follows

$$
H=\frac{m_{p}}{2} \sum_{i=0}^{N+1}\left(\dot{x}_{i}^{2}+\dot{y}_{i}^{2}\right)+\frac{k}{2} \sum_{i=0}^{N+1} y_{i}^{2}-p\left(x_{N+1}-x_{0}\right) .
$$

This then leads to a reasonably natural definition of total energy per link at time $t$, $E_{i}(t)$ for $i=1, \ldots, N$, where we share the kinetic energy of each pivot between the two adjacent links to get

$$
\begin{aligned}
E_{i}(t)=\frac{m_{p}}{4}\left(\dot{x}_{i}^{2}+\dot{y}_{i}^{2}+\dot{x}_{i+1}^{2}+\dot{y}_{i+1}^{2}\right)+\frac{k}{4}\left(y_{i}^{2}+\right. & \left.y_{i+1}^{2}\right)-p\left(x_{i+1}-x_{i}\right) \\
& +\frac{m_{p}}{4}\left(\dot{x}_{0}^{2} \delta_{1, i}+\dot{x}_{N+1}^{2} \delta_{N, i}\right),
\end{aligned}
$$

where $\delta_{i, j}$ is the Kronecker delta symbol and these terms account for the behaviour at the lattice boundaries. Averaging this energy over one breather period gives us the mean energy per link for a breather solution defined by

$$
\bar{E}_{i}=\frac{1}{\tau_{b}} \int_{0}^{\tau_{b}} E_{i}(t) \mathrm{d} t
$$

Figure 6.5 (a) shows a plot of $\log \left(\bar{E}_{i}\right)$ against $i$ for a linearly stable site centred breather in a mechanical lattice with $N=64$. We can see that, firstly, the energy per link in the core centre is several orders of magnitude larger than the energy per link in the tails. Secondly, we can also see that in the core region the energy does localise exponentially. The slight rise in the energy per link for the links at the ends of the lattice seen in Figure 6.5 is due to the end effects of the Kronecker delta terms in the definition of the energy per link, equation (6.2).

To consider the total energies in the core and tail we need to define which links are part of the breather core and which are part of the breather tail. We do this by defining the link numbers $n_{1}$ and $n_{2}\left(n_{2}>n_{1}\right)$ such that links $1, \ldots, n 1$ and $n_{2} \ldots, N$ are part of the tail and links $n_{1}+1, \ldots, n_{2}-1$ form the breather core. We define $n_{1}$ and $n_{2}$ mathematically by using a cut off of $10^{-4}$ on the maximum pivot amplitude:

$$
\begin{aligned}
& n_{1}=\left\{\max n: \max _{t}\left(\left|y_{i}(t)\right|\right)<10^{-4} \text { for all } i \leq n\right\} \quad \text { and } \\
& n_{2}=\left\{\min n: \max _{t}\left(\left|y_{i}(t)\right|\right)<10^{-4} \text { for all } i \geq n\right\} .
\end{aligned}
$$




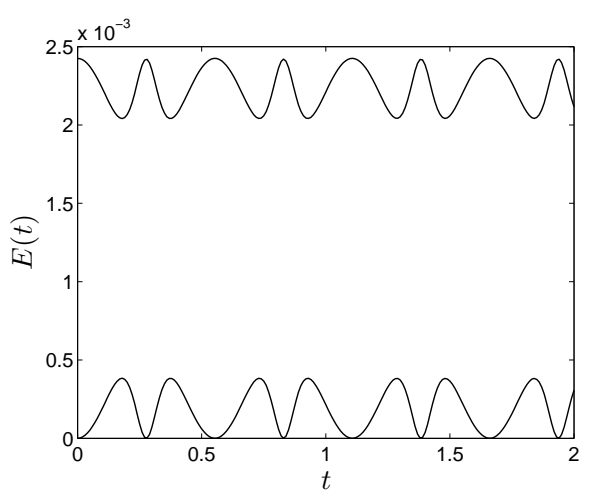

FIG. 6.4. This shows the how the energy of a mechanical breather in a lattice of size $N=64$ with $p=0.3$ and $\omega=0.7$ oscillates between the core of the breather (upper line) and breather tail (lower line). These two energies are defined in equation (6.4).

(a)

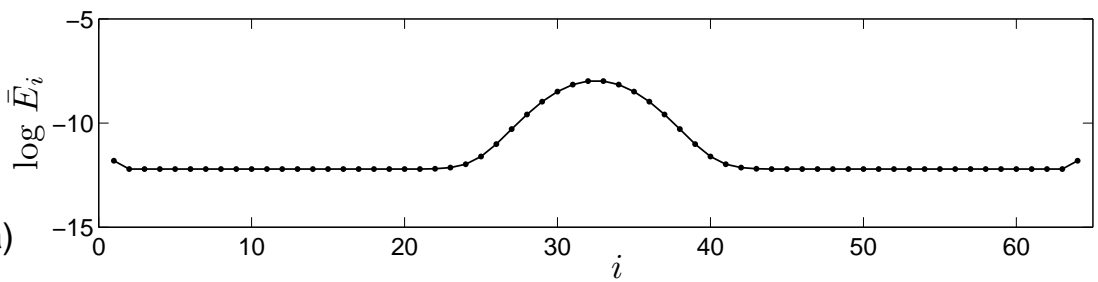

(b)

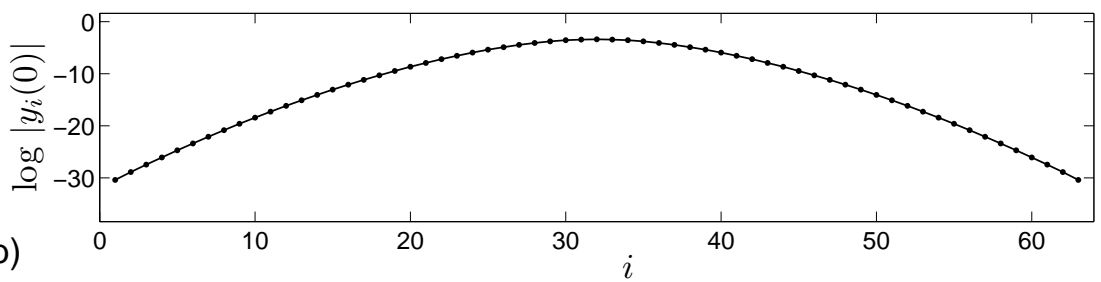

FIG. 6.5. The logarithm of the mean energy per link (see equation 6.2) for the breather of Figure 6.4 is plotted in (a). This shows the exponential localisation of energy in the breather core and the residual energy in the breather tails. The slight increase in link energy at each end of the lattice is due to boundary effects in the definition of $E_{i}(t)$, equation (6.2). In (b) the logarithm of the vertical pivot displacements is shown and we can see that they are at least exponentially localised.

We then have

$$
E_{\text {core }}(t)=\sum_{i=n_{1}+1}^{n_{2}-1} E_{i}(t), \quad \text { and } \quad E_{\text {tail }}(t)=\sum_{i=1}^{n_{1}} E_{i}(t)+\sum_{i=n_{2}}^{N} E_{i}(t) .
$$

Figure 6.4 shows plots of $E_{\text {core }}(t)$ and $E_{\text {tail }}(t)$ for the breather with $N=64$ shown in Figure 6.5. We can see that the energy in the core is more than the energy in the tails and that the tails have only kinetic energy as there is no end-shortening or vertical displacement into the foundation springs in the tails. Even with the large lattice of $N=64$ we can see that the maximum energy, over one breather period, in the breather core is significantly larger that the maximum energy in the tails with $\max _{t} E_{\text {core }}(t) / \max _{t} E_{\text {tail }}(t) \approx 6.3$.

It is interesting to note what happens to these breather solutions as we increase 

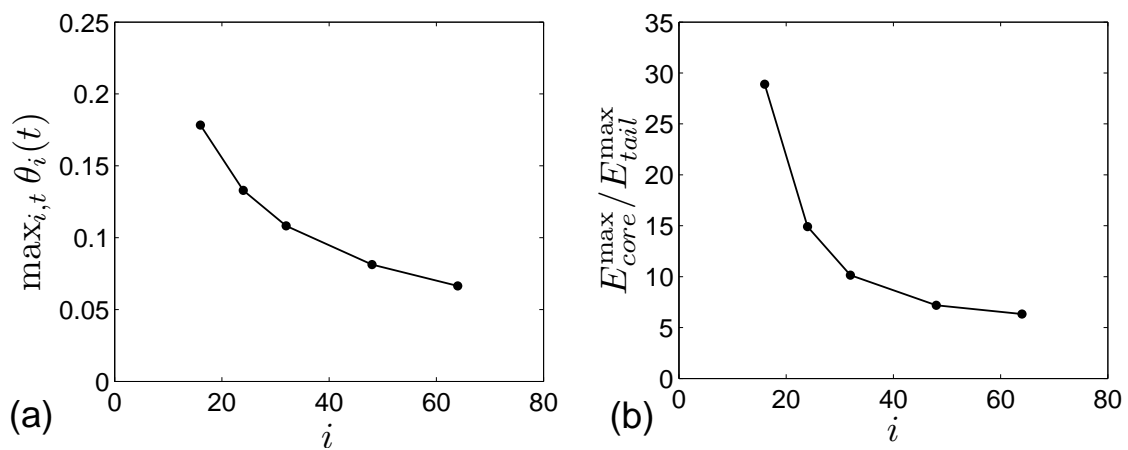

FIG. 6.6. These plots show how the maximum link angle (a) and ratio of the maximum breather core energy, $E_{\text {core }}^{\max }=\max _{t} E_{\text {core }}(t)$, to breather tail energy, $E_{\text {tail }}^{\max }=\max _{t} E_{\text {tail }}(t)$, (b) change as the lattice size $N$ increases. These breather solutions have parameter values $p=0.3$ and $\omega=0.7$.

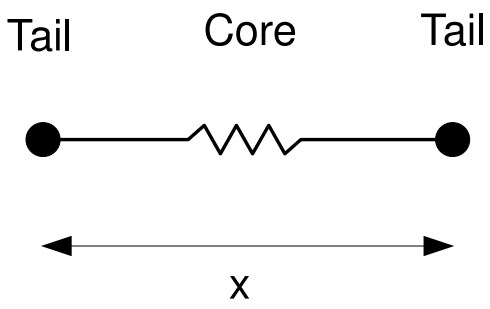

FIG. 6.7. A simplified model of a mechanical breather.

the lattice size $N$ (with all other parameters constant), to see whether the energy in the tails can become larger than the energy in the core. We can see in Figure 6.6 (a) that the amplitude of the breather decreases with increasing lattice size $N$. Figure 6.6 also shows us that the ratio $\max _{t} E_{\text {core }}(t) / \max _{t} E_{\text {tail }}(t)$ also decreases with $N$. But will this ratio ever drop below one? By considering a simplified model of the breather situation we can see that the answer to this question is no.

Figure 6.7 shows a simplified model of a mechanical breather where we consider the two tails as point masses oscillating either side of the core which we consider as a 'black box' spring which acts to make the tails oscillate horizontally. As the tails oscillate in the model of Figure 6.7, since the mechanical system is conservative, their energy moves between the kinetic energy of the tails and potential energy stored in the core. This shows that the energy of the core must, at least, be as large as that of the tails. In fact, the actual breather core 'black box' has its own degrees of freedom which mean that its energy will always be greater than that of the tails.

7. Conclusions. In this paper we have located discrete breathers in the pinned mechanical lattice shown in Figure 1.1. These breathers were found computationally using numerical continuation to follow branches of breather solutions from lattices in which we know they exist to this mechanical lattice. We then went on to discuss the properties of these spatially localised time periodic solutions to the lattice's equations of motion.

A point key to the physical relevance of these breathers is that the maximum displacement of a link in a breather solution is small (for example, a breather with 
$N=32, \omega=0.7 p=0.3$ has $\left.\max _{i, t}\left(\theta_{i}(t)\right) \approx 6.2^{\circ}\right)$ and this reduces as the lattice size $N$ increases. This means that these solutions could be important in structures that allow a small degree of movement, such as bridges, pylons and high-rise buildings. We also find that the breather solutions exist in the mechanical lattice under tension, compression and in the unloaded state. The last of these three states is particularly surprising as the only nonlinearity in the system when it is unloaded is in the inertial terms of the equations of motion.

Another key point in the physical relevance of these mechanical breather solutions is that, in $\S 6.1$, we see that there is a large region in load-frequency parameter space in which these breather solutions are linearly stable. A numerical simulation of a perturbed exact breather solution in the fully nonlinear lattice also indicates that these solutions are nonlinearly stable too.

One feature of the mechanical structure studied here that distinguishes this from lattices in which breather have previously been found is that the rigid links of the lattice mean that vertical movements of the mechanical pivots near the centre of the lattice affect the horizontal movements of the links and pivots at the ends of the lattice. This has the potential to reduce if not remove the key localisation properties of breather solutions. However, in $\S 6.2$ we saw that over the breather core region, both energy and vertical displacement are still exponentially localised. We also saw that the energy of the tails cannot exceed the energy in the breather core no matter how large the lattice.

This paper has focused on the ability of a mechanical lattice system to support spatially localised, time periodic behaviour, or discrete breathers. For localised behaviour to be significant in real structural systems we might also hope that this localisation behaviour can spontaneously appear from apparently random initial conditions. Figure 7.1 presents a glimpse that this is also possible in this mechanical structure, in the form of a spontaneously appearing moving discrete breather (see Figure 7.2). Figure 7.1 shows the results of a long time integration of the mechanical lattice of this paper with random initial conditions (using the energy preserving numerical integrator of Appendix A). Figure 7.1 shows how the energy per link (equation (6.2)) varies with time, and we can clearly see the appearance of spontaneous energy localisation. This provides an interesting glimpse of the additional behaviour one might hope to observe in an experiment on this mechanical lattice.

Acknowledgements. SCG would like to thank EPSRC for a DTA grant. All of the authors would like to thank the anonymous referees for their insightful comments on the first submission of this paper which has led to a significantly improved manuscript.

Appendix A. Generalised RATTLE constraint based formulation. Here we describe a constraint based mathematical model of the mechanical system of Figure 1.1. In this paper we use this model in conjunction with the generalised RATTLE algorithm (see [8, §VI.1.3]) to solve for the time dependence of the mechanical lattice as an IVP whilst preserving the total energy in the numerical solution. This allows us to accurately compare the energy in the core and the tails of the breather and also to get good accurate results on the behaviour during long time simulations.

For this model of the mechanical system we assign each of the $N+1$ pivots (mass $m_{p}$ ) a horizontal, $x$, and vertical, $y$, coordinate. Since we are taking the links to be 


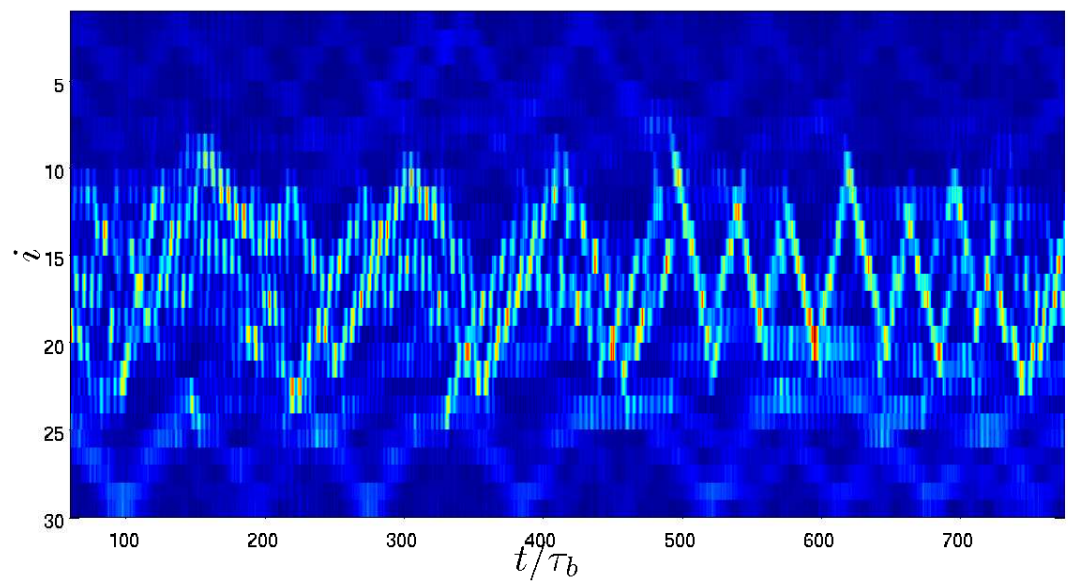

FIG. 7.1. A short snapshot in time of how the energy per link (see equation (6.2)) of the mechanical lattice shown in Figure 1.1 changes over time in the lattice with a set of random initial conditions. Red shows regions of higher energy per link and blue indicates lower energy, clearly showing regions of spontaneous energy localisation.

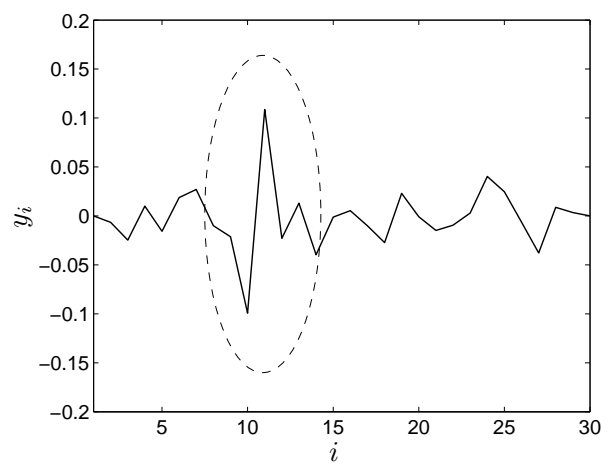

FIG. 7.2. Snapshot at $t=605.3 \tau_{b}$ of the long time integration shown in Figure 7.1. The feature in the lattice circled is, perhaps, an example of a moving discrete breather that is leading to the moving patch of localised energy that appears again and again in Figure 7.1.

mass-less, the kinetic and potential energies of the lattice are then easy to write down:

$$
T(\dot{\mathbf{x}}, \dot{\mathbf{y}})=\frac{m_{p}}{2} \sum_{i=1}^{N+1}\left(\dot{x}_{i}^{2}+\dot{y}_{i}^{2}\right), \quad V(\mathbf{y})=\frac{k}{2} \sum_{i=1}^{N+1} y_{i}^{2}-p\left(x_{N+1}-x_{0}\right) .
$$

These expressions then lead to the Hamiltonian $H(\mathbf{x}, \mathbf{y}, \dot{\mathbf{x}}, \dot{\mathbf{y}})=T(\dot{\mathbf{x}}, \dot{\mathbf{y}})+V(\mathbf{x}, \mathbf{y})$. However, this Hamiltonian does not completely specify our mechanical system. We need to include some constraints to account for the rigid links, vertically fixed boundaries and fixed horizontal lattice centre of mass $(\bar{x})$. We do this by introducing the 
constraint function $g: \mathbb{R}^{2(N+1)} \rightarrow \mathbb{R}^{N+2}$ where

$$
\begin{aligned}
& 0=g_{n} \equiv\left(x_{n+1}-x_{n}\right)^{2}+\left(y_{n+1}-y_{n}\right)^{2}-h^{2} \quad \text { for } \quad n=1, \ldots, N-1 \\
& 0=g_{N} \equiv y_{1} \\
& 0=g_{N+1} \equiv y_{N+1} \\
& 0=g_{N+2} \equiv \frac{1}{N+1} \sum_{n=1}^{N+1} x_{i}-\bar{x} .
\end{aligned}
$$

The generalised RATTLE algorithm described in [8, §VI.1.3] is a numerical integrator for constrained, Hamiltonian dynamical systems that satisfies the constraints exactly at each time step and is also an energy preserving numerical integrator. It is implemented at each time step by solving the following equations

$$
\begin{aligned}
p_{n+\frac{1}{2}} & =p_{n}-\frac{h}{2}\left(H_{q}\left(p_{n+\frac{1}{2}}, q_{n}\right)+g^{\prime}\left(q_{n}\right)^{T} \lambda_{n}\right) \\
q_{n+1} & =q_{n}+\frac{h}{2}\left(H_{p}\left(p_{n+\frac{1}{2}}, q_{n}\right)+H_{p}\left(p_{n+\frac{1}{2}}, q_{n+1}\right)\right) \\
p_{n+1} & =p_{n+\frac{1}{2}}-\frac{h}{2}\left(H_{q}\left(p_{n+\frac{1}{2}}, q_{n+1}\right)+g^{\prime}\left(q_{n+1}\right)^{T} \mu_{n}\right) \\
g\left(q_{n+1}\right) & =0 \\
g^{\prime}\left(q_{n+1}\right) H_{p}\left(p_{n+1}, q_{n+1}\right) & =0 .
\end{aligned}
$$

\section{REFERENCES}

[1] S AubRy, Breathers in nonlinear lattices: Existence, linear stability and quantization, Physica D: Nonlinear Phenomena, 103 (1997), pp. 201-250.

[2] - Discrete breathers: Localization and transfer of energy in discrete Hamiltonian nonlinear systems, Physica D: Nonlinear Phenomena, 216 (2006), pp. 1-30.

[3] S Aubry, G Kopidakis, And V Kadelburg, Variational proof for hard discrete breathers in some classes of Hamiltonian dynamical systems, Discrete and Continuous Dynamical Systems - Series B, 1 (2001), pp. 271-298.

[4] E J Doedel, A R Champneys, T F Fairgrieve, Yu A Kuznetsov, B Sandstede, and $\mathrm{X}$ WANG, Auto97: Continuation and bifurcation software for ordinary differential equations (with Hom Cont). Technical Report, Concordia University, 1997.

[5] G Domokos And P Holmes, Euler's problem, Euler's method, and the standard map; or, the discrete charm of buckling, Journal of Nonlinear Science, 3 (1993), pp. 109-151.

[6] S. Flach AND A. GoRBACH, Computational studies of discrete breathers - from basics to competing length scales, International Journal of Bifurcation and Chaos, 16 (2006), pp. 16451669.

[7] S Flach and C R Willis, Discrete breathers, Physics Reports, 295 (1998), pp. 181-264.

[8] E Hairer, C Lubich, and G Wanner, Geometric Numerical Integration, Springer Series in Computational Mathematics, Springer, 2002.

[9] G W Hunt, R Lawther, and P Providencia E Costa, Finite element modelling of spatially chaotic structures, International Journal for Numerical Methods in Engineering, 40 (1997), pp. 2237-2256.

[10] G James, Centre manifold reduction for quasilinear discrete systems, Journal of Nonlinear Science, 13 (2003), pp. 27-63.

[11] R S MACKAY AND S AUBRY, Proof of existence of breathers for time-reversible or Hamiltonian networks of weakly coupled oscillators, Nonlinearity, 7 (1994), pp. 1623-1643.

[12] J L MARIN AND S AuBRY, Breathers in nonlinear lattices: Numerical calculation from the anticontinuous limit, Nonlinearity, 9 (1996), pp. 1501-1528.

[13] F M Russell, Y Zolotaryuk, and J C EilbeCK, Moving breathers in a chain of magnetic pendulums, Physical Review B, 55 (1997), pp. 6304-6308. 